\title{
Coherent Swing Instability of Power Grids
}

\author{
Yoshihiko Susuki • Igor Mezić • Takashi Hikihara
}

Received: 22 September 2009 / Accepted: 11 December 2010 / Published online: 7 February 2011 (C) The Author(s) 2011. This article is published with open access at Springerlink.com

\begin{abstract}
We interpret and explain a phenomenon in short-term swing dynamics of multi-machine power grids that we term the Coherent Swing Instability (CSI). This is an undesirable and emergent phenomenon of synchronous machines in a power grid, in which most of the machines in a sub-grid coherently lose synchronism with the rest of the grid after being subjected to a finite disturbance. We develop a minimal mathematical model of CSI for synchronous machines that are strongly coupled in a loop transmission network and weakly connected to the infinite bus. This model provides a dynamical origin of CSI: it is related to the escape from a potential well, or, more precisely, to exit across a separatrix in the dynamical system for the amplitude of the weak nonlinear mode that governs the collective motion of the machines. The linear oscillations between strongly coupled machines then act as perturbations on the nonlinear mode. Thus we reveal how the three different mode oscillations-local plant, inter-machine, and inter-area modes - interact to destabilize a power grid. Furthermore, we present a phenomenon of short-term swing dynamics in the New England (NE) 39-bus test system, which is a well-known benchmark model for power grid sta-
\end{abstract}

Communicated by V. Rom-Kedar.

Y. Susuki $(\bowtie) \cdot$ I. Mezić

Department of Mechanical Engineering, University of California, Santa Barbara, CA 93106-5070, USA

e-mail: susuki@ieee.org

Present address:

Y. Susuki

Department of Electrical Engineering, Kyoto University, Katsura, Nishikyo, Kyoto 615-8510, Japan

T. Hikihara

Department of Electrical Engineering, Kyoto University, Katsura, Nishikyo, Kyoto 615-8510, Japan 
bility studies. Using a partial linearization of the nonlinear swing equations and the proper orthonormal decomposition, we show that CSI occurs in the NE test system, because it is a dynamical system with a nonlinear mode that is weak relative to the linear oscillatory modes.

Keywords Power system $\cdot$ Stability $\cdot$ Transient stability $\cdot$ Coupled dynamical systems $\cdot$ Coupled oscillators $\cdot$ Nonlinear dynamics

Mathematics Subject Classification (2000) 37N99 - 70K20 • 93C10

\section{Introduction}

Coupled swing dynamics in a population of synchronous rotating machines are of vital importance for power grid stability. The so-called transient stability analysis is associated with the ability of a power grid to maintain synchronism when subjected to a large disturbance (Kundur 1994; Chiang 1999; IEEE/CIGRE Joint Task Force on Stability Terms and Definitions 2004). Loss of transient stability is recognized as one cause of large blackouts such as the September 2003 blackout in Italy (Corsi and Sabelli 2004; Andersson et al. 2005). Transient stability is mainly governed by oscillations of relative rotor angles between different rotating machines in the short-term regime (0 to 10 seconds (Kundur 1994)) and is mathematically investigated by the so-called nonlinear swing equations (Kundur 1994; Chiang 1999). This mathematical model is similar to equations of motion for coupled mechanical pendulums and is valid because, for a balanced power grid, the dynamics can be described by the relative rotor angles and amplitudes of machine voltages, and in the short-term regime the amplitudes can be assumed to be stationary. Analysis of the swing equations, which especially deals with global structures of phase space far from equilibria, is hence needed for prevention of loss of transient stability (see e.g. Kopell and Washburn 1982; Abed and Varaiya 1984; Salam et al. 1984; Varghese and Thorp 1988; Ueda et al. 1992).

In addition to the above motivation of the stability problem for large-scale power grids, designs of renewable energy sources offer a new situation of swing dynamics and instabilities for small-scale power grids. The notion of a small-scale grid implies that its geographical scale is much smaller than the current nationwide grids. This small-scale feature inevitably results in a power grid in which generation plants and loads are closely coupled. The reasons why the small-scale grid has recently emerged are as follows.

(1) Since most renewable energy sources have smaller outputs than the conventional thermal and nuclear plants, the integration of many renewable sources is inevitable for producing a large amount of energy.

(2) The close coupling of generation plants and loads can contribute to the reduction of loss of energy for transmission and realize an energy-efficient infrastructure.

Examples of such small-scale grids include windfarms and microgrids. A windfarm has many induction generators that operate in parallel and supply the electricity 
to the outside of the farm (see e.g. Null and Archer 2008). A microgrid is defined as an autonomous grid based on dispersed generations (see e.g. Lasseter and Paigi 2004; Hatziargyriou et al. 2007) and has many power sources operating in a closely coupled, parallel mode. Thus, it is fundamentally important to clarify swing dynamics and stability of small-scale power grids for enhancing their performance. This configuration for the small-scale grid is essentially different from the conventional one in transient stability problems, in which many generators and loads are sparsely placed and connected via a complex transmission network.

In this paper, we study an instability phenomenon, which we term the Coherent Swing Instability (CSI), in short-term swing dynamics for multi-machine power grids. CSI is an undesirable phenomenon of synchronous machines in a power grid, in which a group of machines in the grid coherently loses synchronism with the rest of the grid after being subjected to a finite, possibly local disturbance. One goal of our study is to demonstrate swing dynamics that cause a cascade of failures in an interconnected power grid. Blackouts spreading into a large-scale power grid are brought about by a sequence of failures (Andersson et al. 2005). A large-scale grid usually consists of strongly inner-connected grids, called sub-grids, which are coupled to a weak transmission network. The instability studied in this paper describes a failure of one sub-grid caused by the CSI. In Susuki et al. (2010,2011) we show that a sequence of sub-grid failures can be induced by a sequence of CSIs. Thus we show how the sequence of sub-grid failures can be dynamically organized. In this paper, we start from a rudimentary power grid whose dynamics we can study in detail, analytically and numerically, and we expand our study to a more realistic power grid which we study mostly numerically.

CSI is related to three known phenomena in loss of transient stability: local plant mode oscillations, inter-area mode instabilities, and multi-swing instabilities. A local plant mode oscillation is associated with the swinging of a single plant with respect to the grid (Kundur 1994; IEEE/CIGRE Joint Task Force on Stability Terms and Definitions 2004). An inter-area mode oscillation is associated with the swinging of many machines in one part of the grid against machines in other parts (Kundur 1994). Instability of inter-area mode oscillation is defined as the growth of the amplitudes of relative rotor angles as time passes and it implies the separation of a subgroup of machines from the rest of the grid. In Avramovic et al. (1980) the authors extract the inter-area mode, which they call the slow mode, by applying the singular perturbation technique to the linear equations that are derived by linearizing the nonlinear swing equations. In Peponides et al. (1982) the authors apply the singular perturbation technique to the nonlinear swing equations and obtain a set of variables with different time scales that consists of the slow inter-area variable and fast inter-machine ones. For the example in Peponides et al. (1982), the slow inter-area mode is described by the Center-Of-Angle (COA) variables (Athay et al. 1979) or Center-Of-Inertia (COI) variables (Kundur 1994) (see Sect. 4.1 for the definition). Instability mechanisms in the inter-area modes have been studied by various groups of researchers. In Tamura and Yorino (1987) the authors associate parametric resonance in the wellknown Mathieu diagram with long-term (more than 10 seconds) dynamics in multimachine power systems. They use the linearized swing equations with quadratic nonlinear terms and point out the occurrence of sustained oscillations. In Vittal et al. 
(1991), Lin et al. (1996), the authors study the inter-area mode phenomena in terms of the interaction of natural modes in oscillation via quadratic nonlinear terms. They show that the nonlinear modal interaction indicates the onset of inter-area mode phenomena, in which a finite, large initial disturbance results in the separation of a large group of generators from the rest of the system. In Dobson et al. (2001) the author studies the so-called strong modal resonance due to the change of model parameters and associates it with the occurrence of sustained oscillations via Hopf bifurcations. A multi-swing instability is a short-term oscillatory instability and implies the separation of one machine or a group of machines after several swings beyond the first swing. Mechanisms underlying this instability have also been studied. In Vournas et al. (1996) the authors use a detailed model with excitation control and clarify the transient dynamics close to an unstable limit cycle that cause the instability. In Chu (2005) the author reviews lobe dynamics around a tangled separatrix due to periodic forcing and associates it with the occurrence of multi-swing instability.

In this paper, we provide analytical and numerical analysis of CSI, where the phenomenon involves the progression from a local plant mode oscillation, through possibly a multi-swing (or inter-machine mode) oscillation, to the full inter-area mode phenomenon. In this way, we show that these separate phenomena can play a role in the initiation and development of the inter-area mode phenomenon. The contributions of this paper are two: (1) to develop a minimal mathematical model of CSI, based on the notion of instability occurring for general oscillatory systems described in Mezić (2005b, 2006), Eisenhower and Mezić (2007, 2008, 2010), Du Toit et al. (2009), and (2) to explain the instability phenomenon occurring in power grids using the theory of dynamical systems and the model developed. This notion of instability is somewhat broader than the standard definition in dynamical systems (Wiggins 1988), in the sense that it does not happen upon an infinitesimally small perturbation around an equilibrium of the system. However, it encompasses the situation when the system escapes a predefined set around the equilibrium. Thus, the notion of instability that we use here is not local. The model developed here provides a dynamical origin of the non-local instability in power grids as well as a wide range of oscillatory systems.

The organization of this paper is as follows: in Sect. 2 we derive the dynamical model for synchronous machines operating in a loop transmission network connected to the infinite bus. The model explicitly describes both spatial oscillatory modes of the loop network and local dynamics of individual machines with nonlinear power-angle characteristics. In Sect. 3 we provide an example of CSI obtained by numerical simulations of the model. Section 4 is devoted to mathematical analysis of CSI. A change of variables is performed to facilitate the analysis. This reveals the existence of a global mode that governs behavior in the rotor angle of every machine as well as uniform motion of the network, which we call the nonlinear mode. Next we develop a reduced-order, planar dynamical system that describes the behavior of the nonlinear mode and characterizes the CSI. The planar system has one separatrix that depends on both the loop topology and the power-angle characteristics. Analysis of the reduced-order model shows that CSI is related to the escape phenomenon (Thompson and Stewart 2002) or transport phenomenon (Wiggins 1992) in the system describing dynamics of the nonlinear mode, and more precisely, to exit across the separatrix, akin to the study on multi-swing instability (Chu 2005). However, in our description, 
Fig. 1 Rudimentary power grid with the loop topology

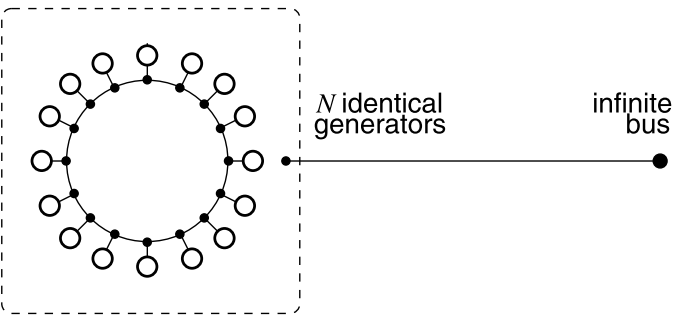

the forcing comes from the other oscillatory modes of the model that are linear and strong compared with the nonlinear mode. Hence we show that CSI occurs as a result of the interplay between grid topology - in the sense of strong interconnections between the machines - and the weak local dynamics of machines. In Sect. 5 we analyze the CSI phenomenon in the New England (NE) 39-bus test system. The NE test system is a well-known benchmark system (Athay et al. 1979) and exhibits coupled swing dynamics of 10 synchronous machines. We present simulations of short-term swing dynamics of synchronous generators in the NE test system using the nonlinear swing equations. By projecting the dynamics onto the phase plane of COA variables, we show that the same mechanism of CSI as the one shown in the simple loop grid emerges. Using a partial linearization of the swing equations and applying the Proper Orthonormal Decomposition (POD) to the data, we show that the reason why CSI happens in the NE test system is again the existence of a nonlinear mode that is weak compared to linear oscillatory modes. This dynamical structure is equivalent to that for the loop case. Thus we suggest that CSI is a phenomenon that occurs for various realistic power grids. Section 6 concludes this paper with a summary and discussion. This paper is a substantially enhanced version of the conference proceedings (Susuki et al. 2008, 2009).

\section{A Mathematical Model for Loop Power Grid}

\subsection{The Swing Equations}

Consider a rudimentary power grid with the loop topology shown in Fig. 1, which we call the loop power grid. Each small circle in Fig. 1 represents a synchronous machine supplying electric power. The grid consists of $N$ small, identical generators, encompassed by the dotted box, which operate in the AC loop network and are connected to the infinite bus. We make five assumptions for the loop grid.

(i) The generators are small, and the transmission lines joining the infinite bus and a generator are much longer than those joining two generators in the loop network. Thus the magnitude of the interaction of an individual generator with the infinite bus is smaller than the magnitude of interaction between any two generators.

(ii) The lengths of transmission lines between the infinite bus and individual generators are identical.

(iii) The lengths of transmission lines between generators are identical.

(iv) The power grid is loss-less. 
(v) The transformer inductance and synchronous reactance of each generator are negligible.

These assumptions enable us to derive a simple mathematical model of CSI, and they can be relaxed substantially while still observing the same phenomenon, as shown in Sect. 5.

Next, we introduce the equations of motion for the power grid. We denote by $\delta_{i}$ the angular position of a rotor with respect to the infinite bus of generators labeled with integer values $i=1, \ldots, N$. The non-dimensional deviation of rotor speed in generator $i$ relative to the system angular frequency (normally $2 \pi \times 50 \mathrm{rad} / \mathrm{s}$ or $2 \pi \times$ $60 \mathrm{rad} / \mathrm{s}$ ) is denoted by $\omega_{i}$. The short-term dynamics of generator $i$ are represented by the nonlinear swing equations (Kundur 1994)

$$
\frac{\mathrm{d} \delta_{i}}{\mathrm{~d} t}=\omega_{i}, \quad \frac{\mathrm{d} \omega_{i}}{\mathrm{~d} t}=p_{\mathrm{m}}-b \sin \delta_{i}+b_{\mathrm{int}}\left\{\sin \left(\delta_{i-1}-\delta_{i}\right)-\sin \left(\delta_{i}-\delta_{i+1}\right)\right\} .
$$

The loop topology of the generators induces the following conditions:

$$
\delta_{0}(t)=\delta_{N}(t), \quad \delta_{N+1}(t)=\delta_{1}(t) .
$$

The parameters $p_{\mathrm{m}}, b$, and $b_{\text {int }}$ are constant in time and are in per-unit system: see Appendix A. The constant $p_{\mathrm{m}}$ is the mechanical input power to generator $i, b$ is the critical transmission power between the infinite bus and generator $i$, and $b_{\text {int }}$ is the critical transmission power between generators $i$ and $i+1$. The constants $b$ and $b_{\text {int }}$ are inversely proportional to the lengths of the associated AC transmission lines.

\subsection{The Mathematical Model}

We now simplify (1) in order to construct a mathematical model of the phenomenon of interest, where the differences between individual angular positions stay small for all time. Note that there are trajectories of the system (1) that stay on a two-dimensional invariant manifold $\mathcal{I}$ in $2 N$-dimensional phase space of the system, defined by the $2(N-1)$ constraints $\delta_{i}=\delta_{i+1}$ and $\omega_{i}=\omega_{i+1}$ for $i=1, \ldots, N-1$ (see Mezić 2005b). These are trajectories with the common initial conditions $\delta_{i}(0)$ and $\omega_{i}(0)$ for all generators. We investigate the dynamics of the system (1) close to this invariant manifold. This is equivalent to the condition that the differences $\delta_{i}(t)-\delta_{i+1}(t)$ and $\omega_{i}(t)-\omega_{i+1}(t)$ for $i=1, \ldots, N-1$ are sufficiently small. This allows us to use the first-order approximation of the sinusoidal coupling term $b_{\text {int }}\left\{\sin \left(\delta_{i-1}-\delta_{i}\right)-\sin \left(\delta_{i}-\delta_{i+1}\right)\right\}$ and to derive the following system:

$$
\frac{\mathrm{d} \delta_{i}}{\mathrm{~d} t}=\omega_{i}, \quad \frac{\mathrm{d} \omega_{i}}{\mathrm{~d} t}=p_{\mathrm{m}}-b \sin \delta_{i}+b_{\text {int }}\left\{\left(\delta_{i-1}-\delta_{i}\right)-\left(\delta_{i}-\delta_{i+1}\right)\right\} .
$$

The system (3) is an $N$-degree-of-freedom Hamiltonian system with the Hamiltonian function $\mathcal{H}\left(\delta_{1}, \ldots, \delta_{N}, \omega_{1}, \ldots, \omega_{N}\right)$ :

$$
\mathcal{H}=\sum_{i=1}^{N} \frac{\omega_{i}^{2}}{2}-\sum_{i=1}^{N}\left\{p_{\mathrm{m}} \delta_{i}+b \cos \delta_{i}-\frac{b_{\mathrm{int}}}{2}\left(\delta_{i-1}-\delta_{i}\right)^{2}\right\} .
$$


In (3) the linear term is an approximation of the sinusoidal characteristics in power transfer between nearest generators. This approximation is physically valid under the above assumption (i) that the length of transmission lines between nearest generators is much shorter than that between the infinite bus and individual generators. That is, $b_{\text {int }} \gg b$, and the strong local coupling keeps the connected generators in the loop network close in angular position and speed deviation. Furthermore, the term $p_{\mathrm{m}}-b \sin \delta_{i}$ represents the nonlinear power-angle characteristics of each generator connected to the infinite bus and suggests an inherent possibility of loss of synchronism with the infinite bus. This nonlinearity is weak for the above assumption (i) of small generators, that is, $p_{\mathrm{m}}, b \ll b_{\text {int }}$.

The dynamical model (3) contains the terms that can account for the two causes of loss of transient stability that have been discussed in Kundur (1994) from a phenomenological viewpoint. First, in large-scale power grids, loss of transient stability may be the result of the superposition of several modes of oscillation, causing large excursion of angular position beyond the first swing. The term $b_{\text {int }}\left\{\left(\delta_{i-1}-\delta_{i}\right)-\left(\delta_{i}-\delta_{i+1}\right)\right\}$ in (3) can cause such linear spatial oscillatory modes of the loop network (see Sect. 4.2). Second, the multi-swing instability can be related to the collective escape of generators from the potential well, defined by the nonlinear terms $p_{\mathrm{m}}-b \sin \delta_{i}$ in (3) (see Sect. 4.4). However, both of these effects are necessary to produce the instability phenomenon we describe in the next section. To see this, consider these effects separately. If the nonlinear interaction with the infinite bus is removed, no instability is possible, because the motion is a superposition of linear modes. On the other hand, if there are no linear coupling terms, then every oscillator is stable inside its own potential well. Thus, the instability phenomenon that we explain in this paper is due to both the effects of a strong linear coupling and a weak local nonlinearity.

The model (3) is an approximation of the dynamics of the original swing equations (1). Basically the model is valid under the condition that the differences between individual angular positions stay small for all time; mathematically, a trajectory of (3) stays in the neighborhood of the invariant manifold $\mathcal{I}$ for all time. Here, due to the presence of strong linear coupling in (3), many of the trajectories departing far from $\mathcal{I}$ quickly approach $\mathcal{I}$ as time goes on. One example of this phenomenon is given in the next section: see Figs. 2(a) and (b). Thus we expect that analysis of (3) gives a clue to the problem on short-term swing stability in the loop power grid. Sections 3 and 4 are devoted to the analysis of (3).

\section{The Phenomenology of Coherent Swing Instability}

In this section we present numerical simulations of coupled swing dynamics represented by (3) and discuss the properties of the phenomenon that we name the Coherent Swing Instability (CSI). The parameter settings for (3) used throughout most of this section are

$$
p_{\mathrm{m}}=0.95, \quad b=1, \quad b_{\text {int }}=100, \quad N=20 .
$$

The values of $p_{\mathrm{m}} / b_{\text {int }}=0.0095$ and $b / b_{\text {int }}=0.01$ are chosen to satisfy the above physical assumptions that imply a strong linear coupling of generators and a weak 


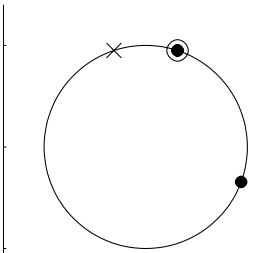

(a) $t=0$

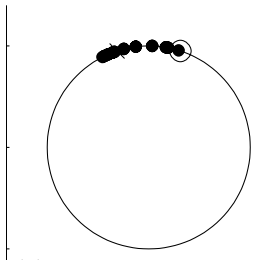

(e) $t=10$

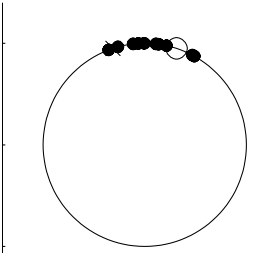

(i) $t=30$

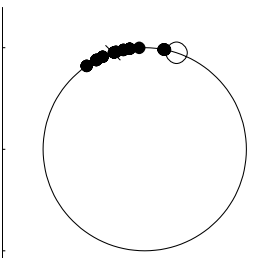

(m) $t=42$

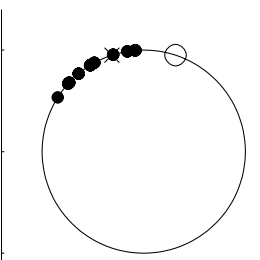

(q) $t=46$

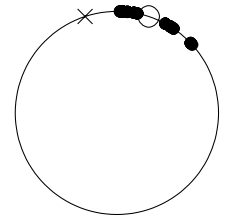

(b) $t=1$

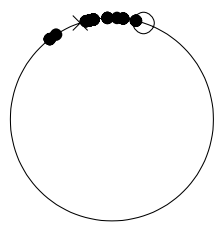

(f) $t=15$

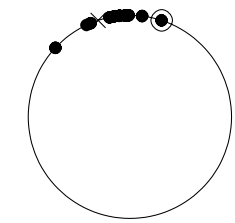

(j) $t=35$

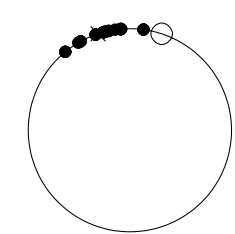

(n) $t=43$

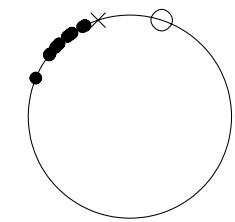

(r) $t=47$

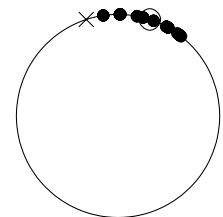

(c) $t=2$

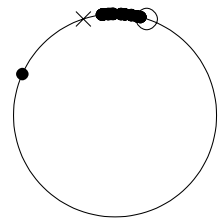

(g) $t=20$

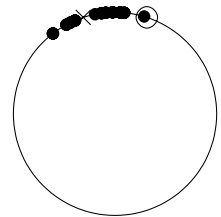

(k) $t=40$

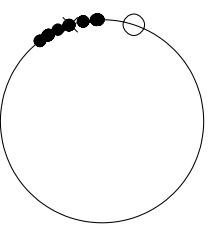

(o) $t=44$

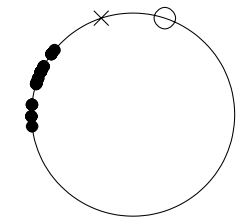

(s) $t=48$

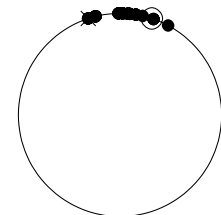

(d) $t=5$

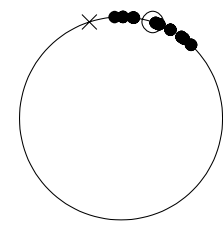

(h) $t=25$

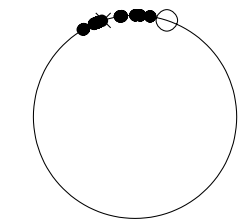

(I) $t=41$

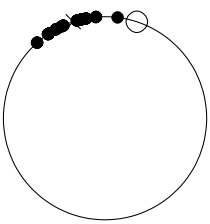

(p) $t=45$

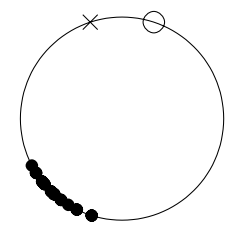

(t) $t=49$

Fig. 2 Sequential snapshots of angular position $\delta_{i}(t)$ for coupled swing dynamics by the local disturbance. Solid points on the circle denote the angular positions $\delta_{i}$ of $N$ generators and move according to (3) from the initial condition (6). At the initial time (a), generator $N / 2$ is disturbed, and its initial position $\delta_{N / 2}(0)$ is denoted by the lower solid point, while all the other generators are at the elliptic equilibrium $\delta_{i}=\delta_{\mathrm{c}}$ denoted by the circle $\circ$. The symbol $\times$ on the circle denotes the position of the hyperbolic equilibrium $\delta_{i}=\pi-\delta_{\mathrm{c}}$

local nonlinearity governing the dynamics of each individual generator. We also study the variation of the observed phenomenon with the mechanical input power $p_{\mathrm{m}}$, the strength of interconnections $b_{\mathrm{int}}$, and the number of generators $N$. All numerical 
integrations of the Hamiltonian system (3) in this paper were performed using the symplectic integrator (Yoshida 1990).

\subsection{Basic Simulation}

Now we describe a basic scenario of coupled swing dynamics and instabilities. Note that if the condition $p_{\mathrm{m}}<b$ holds, there are two global equilibria of (3) at $\left(\delta_{i}, \omega_{i}\right)=$ $\left(\delta_{\mathrm{c}}=\sin ^{-1}\left(p_{\mathrm{m}} / b\right), 0\right)$, which is a global energy minimum of the elliptic type, and $\left(\pi-\delta_{\mathrm{c}}, 0\right)$ of the hyperbolic type. Let us consider a local disturbance given by the following set of initial conditions, close to the elliptic equilibrium:

$$
\left(\delta_{i}(0), \omega_{i}(0)\right)= \begin{cases}(-0.352,0) & \text { for } i=N / 2 \\ \left(\delta_{\mathrm{c}}, 0\right) & \text { for } i \neq N / 2 .\end{cases}
$$

Physically, the generator $N / 2$ is locally disturbed, while the other generators are at their steady states, that is, the elliptic equilibrium denoted by the circle $\circ$ in Fig. 2. The choice of the generator disturbed initially does not depend on the following numerical results, because the system (3) has translational invariance along the loop network. Figure 2 shows sequential snapshots of angular positions $\delta_{i}$ for coupled swing dynamics by the local disturbance. The solid points on the circle denote the angular positions of $N$ generators. At the initial time in Fig. 2(a), generator $N / 2$ is disturbed, and its initial position $\delta_{N / 2}(0)$ is denoted by the lower solid point, while all the other generators are at the same position on the circle, denoted by the upper solid point. In the snapshots shown in Figs. 2(b)-(j), the angular positions of $N$ generators are in an intermediate regime of linear oscillations around their steady states (these snapshots are compared to Fig. 4(a) where the average angle vs. time is plotted). These oscillations are bounded and essentially linear. However, in Figs. 2(j)-(q), they start to show coherent growth. Finally, they grow unbounded in Figs. 2(q)-(t). The unbounded growth indicates that all the generators lose synchronism with the infinite bus. This is the phenomenon that we call the Coherent Swing Instability (CSI). It represents a dynamic transition from a localized perturbation that initially causes linear multi-swing (or inter-machine mode) oscillations and ultimately leads to coherent loss of synchronism of the whole group of generators with the infinite bus, that is, instability of the inter-area mode oscillation.

Note that in Fig. 2 we plot $t$ in units of $\sqrt{2 H / \omega_{\mathrm{b}}}$ where $H$ is the per-unit time constant of each machine, and $\omega_{\mathrm{b}}$ the system angular frequency: see Appendix A. For $H=10 \mathrm{~s}$ and $\omega_{\mathrm{b}}=2 \pi \times(60 \mathrm{~Hz}), t=50$ is equal to $12 \mathrm{~s}$ in real time. Hence the onset time of instability in Fig. 2 is within the short-term regime. Thus, we have discussed the dynamical terms that lead to linear oscillations and (nonlinear) loss of coherence at the end of the previous section, and we have connected them to descriptions of various types of instabilities described in the literature on power systems.

\subsection{Robustness Analysis}

Local disturbances, different from (6), cause instability phenomena with time duration different from that in Fig. 2. Figure 3 plots the destabilization time $t_{\text {inst }}$, which is the time it takes for the collective coordinate-in this case the average of all the 
Fig. 3 Numerical results for time and local disturbance needed for coherent swing instability: destabilization time plotted against initial condition $\delta_{N / 2}$ under $b=1$. Figure (a) is for various values $p_{\mathrm{m}}$ of the mechanical input power with $b_{\text {int }}=100$ and $N=20$.

Figure (b) is for various strengths $b_{\text {int }}$ of the loop network with $p_{\mathrm{m}}=0.95$ and $N=20$, and (c) for various numbers $N$ of generators with $p_{\mathrm{m}}=0.95$ and $b_{\text {int }}=100$. The solid vertical line denotes the position of elliptic equilibrium, and the broken line denotes the position of the hyperbolic saddle. (d) The number of machines, $N$, vs. minimum amplitude of perturbation, $\delta_{N / 2}$ and (e) the number of machines, $N$, vs. minimum perturbation energy per machine. The data are obtained with $p_{\mathrm{m}}=0.95$, $b=1$, and $b_{\text {int }}=100$
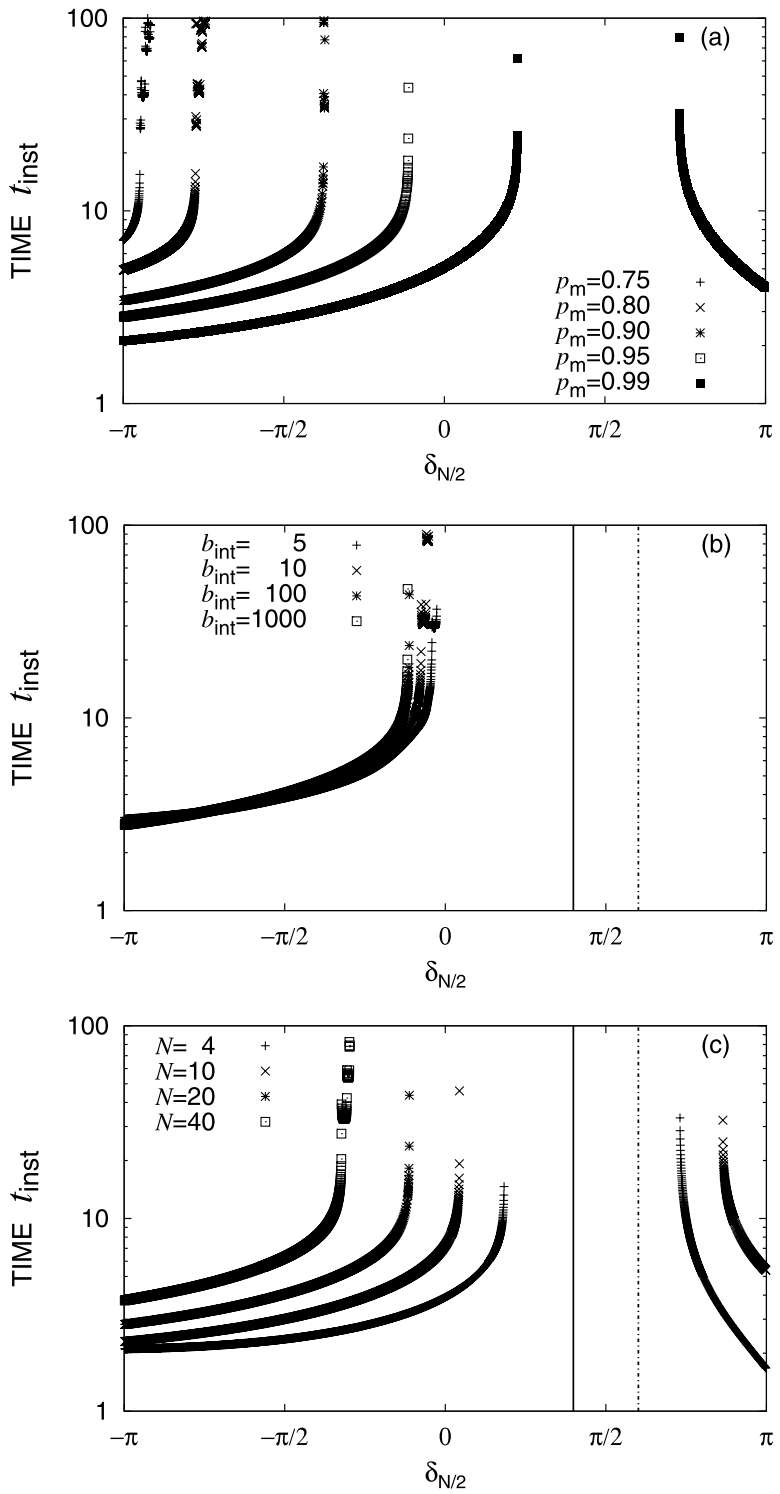

angles $\delta_{i}$ (see Sect. 4) - to reach the position of the (hyperbolic) saddle. This time is an indicator of the amount of time that it takes to reach CSI. In Fig. 3 we show the results of numerical simulations that demonstrate the robustness of the observed CSI phenomenon. On the horizontal axis we plot the initial angular position of disturbance for generator $N / 2$. This angular position parameterizes the total energy of the disturbance since there is no kinetic energy for the initial disturbance. If no instability is observed during the time interval $[0,100)$ we do not plot a point corresponding to the initial disturbance. In Fig. 3(a) we observe that (1) there is a finite initial condition 
Fig. 3 (Continued)
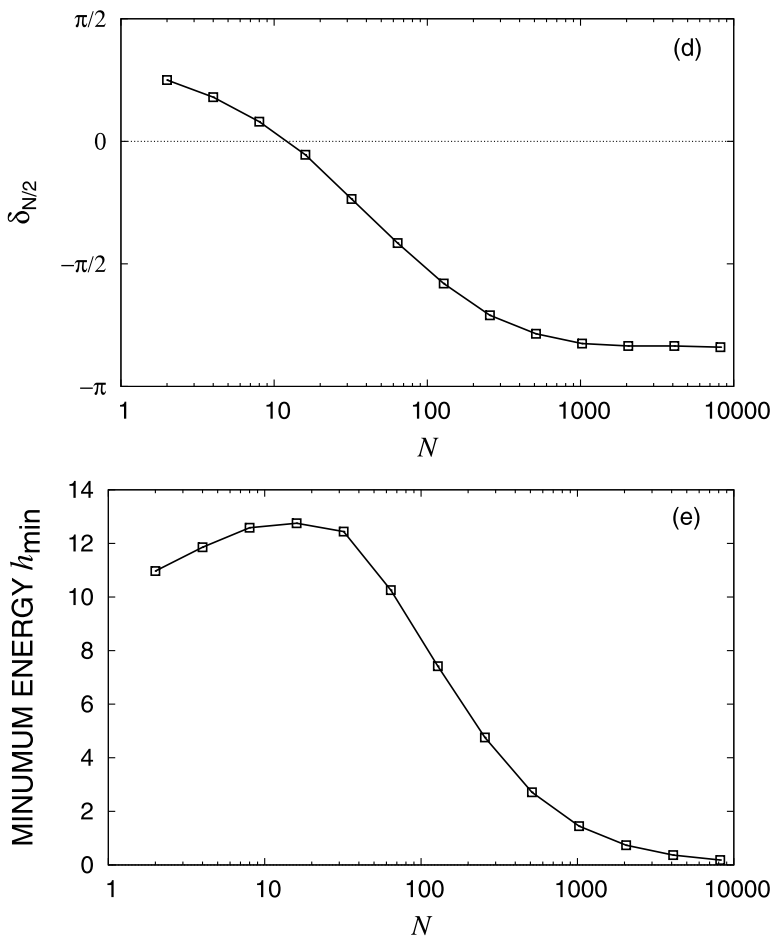

$\delta_{N / 2}$ for which CSI happens for all mechanical input powers $p_{\mathrm{m}}$; that (2) the minimum initial $\delta_{N / 2}$ to cause CSI becomes large with the decrease of $p_{\mathrm{m}}$; and that (3) for $p_{\mathrm{m}}<0.75$ we do not see the instability in the range of initial conditions. The setting of small $p_{\mathrm{m}}$ is interpreted as a light loading condition of generators. Thus, under the conditions in this study, we can say that CSI happens at relatively heavy loading conditions under which $p_{\mathrm{m}}$ is close to the critical transmission power $b$, which is equal to the rating of individual generators (see Appendix A). In addition, the range of $\delta_{N / 2}$ in which instability is not observed becomes narrow with the increase of $p_{\mathrm{m}}$. This is valid because at the limit of $p_{\mathrm{m}}=1$ the two global equilibria disappear in the Hamiltonian saddle-node bifurcation, and thus any initial condition causes instability. In Fig. 3(b) we see that the amplitude of the minimum perturbation $\delta_{N / 2}$ that causes CSI becomes slightly smaller with the decrease of the interconnection strength $b_{\text {int }}$, and that it saturates with $b_{\text {int }}>100$. This implies that sufficiently strong interconnections do not affect the occurrence of CSI qualitatively. In Fig. 3(c) we see that the amplitude of perturbation that causes CSI increases as the number $N$ of generators increases, as would be expected from the intuition that more energy is needed to destabilize a larger number of machines. In addition, from this figure we can see that with the decrease of $N$, the range of $\delta_{N / 2}$ in which instability is not observed is getting closer to the range between the two vertical lines, that is, the closed interval $\left[\delta_{\mathrm{c}}, \pi-\delta_{\mathrm{c}}\right]$. This is a natural result, because the limit of $N=1$ implies the grid of a single machine connecting to the infinite bus, and the instability of single machine does not occur if the initial condition for angular position is placed within the above interval (note that we need the initial condition of zero rotor speed difference). 
From these numerical simulations, we contend that the observed CSI is quite robust to changes in parameters $p_{\mathrm{m}}, b_{\text {int }}$, and $N$.

Figures 3(d) and (e) show numerical results for time and local disturbance needed for CSI. The data are obtained under the parameter settings of $p_{\mathrm{m}}=0.95, b=1$, and $b_{\text {int }}=100$, which are used for Fig. 3(c). The minimum energy per machine, $h_{\min }\left(\delta_{N / 2}, N\right)$, quantifies the minimum energy added by local disturbance to cause CSI and is defined as follows:

$$
h_{\min }=\frac{1}{N}\left\{-\left(p_{\mathrm{m}} \delta_{N / 2}+b \cos \delta_{N / 2}\right)+\left(p_{\mathrm{m}} \delta_{\mathrm{c}}+b \cos \delta_{\mathrm{c}}\right)+b_{\text {int }}\left(\delta_{N / 2}-\delta_{\mathrm{c}}\right)^{2}\right\} .
$$

In Fig. 3(d) we see that the minimum amplitude of perturbation that induces CSI converges to a constant value as $N$ increases. In Fig. 3(e) we see that the minimum energy reaches a maximum value at around $N=20$, gradually decreases as the increase of $N$, and finally converges to zero. The convergence is valid in the definition of $h_{\min }\left(\delta_{N / 2}, N\right)$ if the minimum amplitude of perturbation $\delta_{N / 2}$ remains finite as $N$ goes to infinite. The global maximum of $h_{\text {min }}$ suggests that if we regard the minimum energy per machine as a quantitative measure of transient stability in the loop grid, the increase of the number of machines and interconnections does not necessarily contribute to the enhancement of transient stability and the robust operation of the loop grid.

\section{A Dynamical Mechanism of Coherent Swing Instability}

This section is devoted to mathematical analysis of the CSI shown in Fig. 2. The analysis relies on transformations into action-angle variables for multi-degree-offreedom Hamiltonian systems (Arnold 1989). We first describe the dynamics in collective variables that are averages of individual variables. We then derive a planar dynamical system that describes collective dynamics or dynamics of the nonlinear mode of oscillation. Analysis of this planar system makes it clear that the CSI occurs as a result of the interplay between grid topology and local dynamics.

\subsection{Collective Dynamics}

The collective-phase variable $\delta$ and its time derivative $\omega$ are defined as

$$
\delta=\frac{1}{N} \sum_{i=1}^{N} \delta_{i}, \quad \omega=\frac{\mathrm{d} \delta}{\mathrm{d} t}=\frac{1}{N} \sum_{i=1}^{N} \omega_{i} .
$$

The variables are well known in power grid stability analysis as the COA (Center-OfAngle) or COI (Center-Of-Inertia) variables (Athay et al. 1979; Kundur 1994). Time evolution of these variables, called collective dynamics, of the loop grid is given by the equations

$$
\frac{\mathrm{d} \delta}{\mathrm{d} t}=\omega, \quad \frac{\mathrm{d} \omega}{\mathrm{d} t}=p_{\mathrm{m}}-\frac{b}{N} \sum_{i=1}^{N} \sin \delta_{i} .
$$


Fig. 4 Collective dynamics of coupled swings for the local disturbances $\delta_{N / 2}(0)=0$ and -0.352 : (a) $t-\delta$ curves and (b) trajectories in the $\delta-\omega$ plane
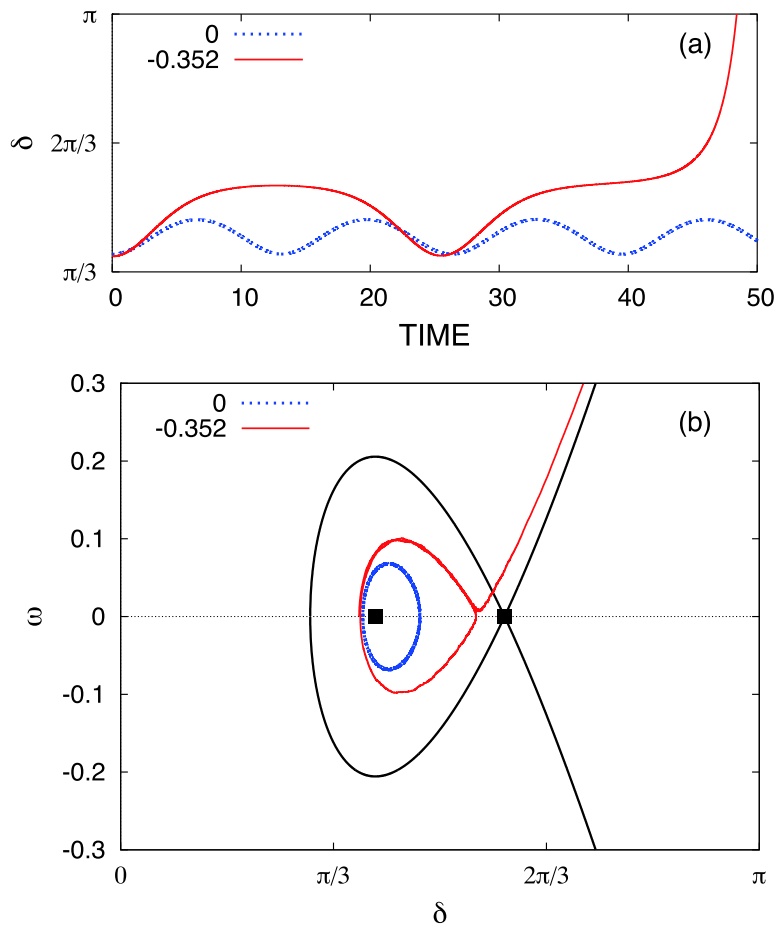

Figure 4 shows two examples of the time evolution of the collective-phase variable $\delta$ and trajectory in the $\delta-\omega$ plane. The solid line is for the initial condition $\delta_{N / 2}(0)=$ -0.352 and corresponds to the CSI in Fig. 2. The broken line is for $\delta_{N / 2}(0)=0$ and does not lead to any instability. The two boxes ( $\boldsymbol{\square})$ represent the equilibria of the following system:

$$
\frac{\mathrm{d} \delta}{\mathrm{d} t}=\omega, \quad \frac{\mathrm{d} \omega}{\mathrm{d} t}=p_{\mathrm{m}}-b \sin \delta .
$$

That is, the system represents the local dynamics of a single generator and is equivalent to equations describing the motion of a mathematical pendulum with external constant torque acting on it. It also describes the dynamics on the two-dimensional invariant manifold $\mathcal{I}$. The variables $(\delta, \omega)$ are regarded as the local coordinates of this manifold. The system (10) with $\left|p_{\mathrm{m}} / b\right|<1$ has the saddle point $\left(\pi-\delta_{\mathrm{c}}, 0\right)$ connected to itself by a homoclinic orbit $\Gamma_{0}$, as drawn in Fig. 4(b).

Now we compare the dynamics of the full system (3) projected onto the $\delta-\omega$ plane with the dynamics of the system (10). For the solid trajectory showing the divergence in Fig. 4(b), the intermediate linear oscillations in Figs. 2(b)-(j) appear as a trapped motion inside $\Gamma_{0}$, and the final coherent growth in Figs. 2(j)-(q) appears as a divergence motion over $\Gamma_{0}$. Trajectories of the system (10) are bounded oscillations (librations) inside $\Gamma_{0}$ and never diverge, or always diverge outside of it. Therefore, the homoclinic orbit $\Gamma_{0}$ defines a boundary of stable region, and we say that CSI occurs when a trajectory projected onto the $\delta-\omega$ plane starts inside the region defined by $\Gamma_{0}$, escapes from it, and diverges to infinity. In this section, we develop a reduced-order, 
planar (two-dimensional), non-autonomous dynamical system that can describe the process of CSI.

\subsection{Mode Decomposition}

By setting $p_{\mathrm{m}}=b=0$, oscillators represented by the linear system derived from (3) can be decomposed into a superposition of eigenmode oscillations of the loop grid. Any motion of the linear system is represented as a superposition of eigenmodes with a finite number of eigenfrequencies. The eigenfunctions $\left\{e_{i j}\right\}_{i, j=1}^{N}$ and eigenfrequencies $\left\{\Omega_{j}\right\}_{j=1}^{N}$ of eigenmodes are given as follows (see e.g. Forest et al. 1992):

$$
e_{i j}=\sqrt{\frac{2}{N}} \cos \left(\frac{2 \pi i j}{N}+\frac{\pi}{4}\right), \quad \Omega_{j}=2 \sqrt{\left|b_{\mathrm{int}}\right|}\left|\sin \frac{\pi j}{N}\right|,
$$

where $i$ is the index of generator, and $j$ is the mode number. These formulas make it possible to investigate (3) in modal variables and action-angle variables.

Now we introduce two types of variable transformations and associate the collective variables $\delta$ and $\omega$ with dynamics of the full system. The first set of variables $\left(u_{j}, v_{j}\right)(j=1, \ldots, N)$ is defined as

$$
u_{j} \triangleq \sum_{i=1}^{N} e_{i j} \delta_{i}, \quad v_{j} \triangleq \sum_{i=1}^{N} e_{i j} \omega_{i}
$$

where $\left(u_{j}, v_{j}\right)$ are the variables of $j$ th spatial mode, called $j$ th modal variables. The Hamiltonian $\mathcal{H}$ can be written in new coordinates $\left(u_{1}, \ldots, u_{N}, v_{1}, \ldots, v_{N}\right)$ as

$$
\mathcal{H}=\sum_{j=1}^{N} \frac{1}{2}\left(v_{j}^{2}+\Omega_{j}^{2} u_{j}^{2}\right)-\sum_{i=1}^{N}\left\{p_{\mathrm{m}} \sum_{j=1}^{N} e_{i j} u_{j}+b \cos \left(\sum_{j=1}^{N} e_{i j} u_{j}\right)\right\} .
$$

The second set of variables we introduce are the action-angle ones (Arnold 1989). It should be noted that the eigenfrequency $\Omega_{N}$ of the $N$ th mode is equal to zero, in other words, the $N$ th mode is non-oscillatory under $p_{\mathrm{m}}=b=0$. We call it the nonlinear mode, because the behavior of the mode under $p_{\mathrm{m}} \neq 0$ and $b \neq 0$ is described by nonlinear differential equations similar to the system (10) (see (16)). Define the variables $\left(I_{j}, \theta_{j}\right)(j=1, \ldots, N-1)$ by

$$
u_{j}=\sqrt{\frac{2 I_{j}}{\Omega_{j}}} \sin \theta_{j}, \quad v_{j}=\sqrt{2 I_{j} \Omega_{j}} \cos \theta_{j},
$$

where $\left(I_{j}, \theta_{j}\right)$ are called the action-angle variables. The Hamiltonian $\mathcal{H}$ becomes

$$
\begin{aligned}
\mathcal{H}= & \frac{1}{2} v_{N}^{2}+\sum_{j=1}^{N-1} I_{j} \Omega_{j}-\sum_{i=1}^{N}\left\{p_{\mathrm{m}} \sum_{j=1}^{N-1} e_{i j} \sqrt{\frac{2 I_{j}}{\Omega_{j}}} \sin \theta_{j}+p_{\mathrm{m}} e_{i N} u_{N}\right. \\
& \left.+b \cos \left(\sum_{j=1}^{N-1} e_{i j} \sqrt{\frac{2 I_{j}}{\Omega_{j}}} \sin \theta_{j}+e_{i N} u_{N}\right)\right\} .
\end{aligned}
$$


As a result of the variable transformations, we obtain the following equations:

$$
\left.\begin{array}{rl}
\frac{\mathrm{d} u_{N}}{\mathrm{~d} t} & =v_{N}, \\
\frac{\mathrm{d} v_{N}}{\mathrm{~d} t} & =p_{\mathrm{m}} \sqrt{N}-b \frac{1}{\sqrt{N}} \sum_{i=1}^{N} \sin \left(\sum_{j=1}^{N-1} e_{i j} \sqrt{\frac{2 I_{j}}{\Omega_{j}}} \sin \theta_{j}+e_{i N} u_{N}\right),
\end{array}\right\}
$$

and

$$
\left.\begin{array}{l}
\frac{\mathrm{d} I_{n}}{\mathrm{~d} t}=b \sqrt{\frac{2 I_{n}}{\Omega_{n}}} \cos \theta_{n} \sum_{i=1}^{N} e_{i n} \sin \left(\sum_{j=1}^{N-1} e_{i j} \sqrt{\frac{2 I_{j}}{\Omega_{j}}} \sin \theta_{j}+e_{i N} u_{N}\right), \\
\frac{\mathrm{d} \theta_{n}}{\mathrm{~d} t}=-\Omega_{n}-b \frac{\sin \theta_{n}}{\sqrt{2 I_{n} \Omega_{n}}} \sum_{i=1}^{N} e_{i n} \sin \left(\sum_{j=1}^{N-1} e_{i j} \sqrt{\frac{2 I_{j}}{\Omega_{j}}} \sin \theta_{j}+e_{i N} u_{N}\right),
\end{array}\right\}
$$

where $n=1, \ldots, N-1$. The interaction between the modes is weak because of $p_{\mathrm{m}}, b \ll b_{\text {int }}$. That is, CSI occurs in a system consisting of the nonlinear mode and $N-1$ oscillatory eigenmodes coupled via weak nonlinear terms.

Here we show that the dynamics of the nonlinear mode derived in the previous subsection are equivalent to the dynamics in the collective variables $(\delta, \omega)$. Because $e_{i N}=1 / \sqrt{N}$ for all $i$, the $N$ th modal variables $\left(u_{N}, v_{N}\right)$ are re-written as

$$
u_{N}=\frac{1}{\sqrt{N}} \sum_{i=1}^{N} \delta_{i}, \quad v_{N}=\frac{1}{\sqrt{N}} \sum_{i=1}^{N} \omega_{i} .
$$

Using $u_{N}=\delta \sqrt{N}$ and $v_{N}=\omega \sqrt{N}$ we re-write (16) as follows:

$$
\left.\begin{array}{l}
\frac{\mathrm{d} \delta}{\mathrm{d} t}=\omega, \\
\frac{\mathrm{d} \omega}{\mathrm{d} t}=p_{\mathrm{m}}-\frac{b}{N} \sum_{i=1}^{N} \sin \left(\sum_{j=1}^{N-1} e_{i j} \sqrt{\frac{2 I_{j}}{\Omega}} \sin \theta_{j}+\delta\right) .
\end{array}\right\}
$$

Now, by direct calculation, we have the following orthonormal property of the eigenvectors $\left\{e_{i j}\right\}$ defined in (11):

$$
\sum_{j=1}^{N} e_{k j} e_{i j}= \begin{cases}1 & k=i \\ 0 & k \neq i\end{cases}
$$

By multiplying both sides of $u_{j}$ in (12) by $e_{k j}$ and summing the resulting equations of $u_{j}$ over $j$, we have

$$
\sum_{j=1}^{N} e_{k j} u_{j}=\sum_{j=1}^{N} \sum_{i=1}^{N} e_{k j} e_{i j} \delta_{i}
$$


Fig. 5 Collective dynamics of coupled swings for the first mode disturbance with different strengths $c=1.7,1.745,1.8$ :

(a) $t-\delta$ curves and

(b) trajectories in the $\delta-\omega$ plane
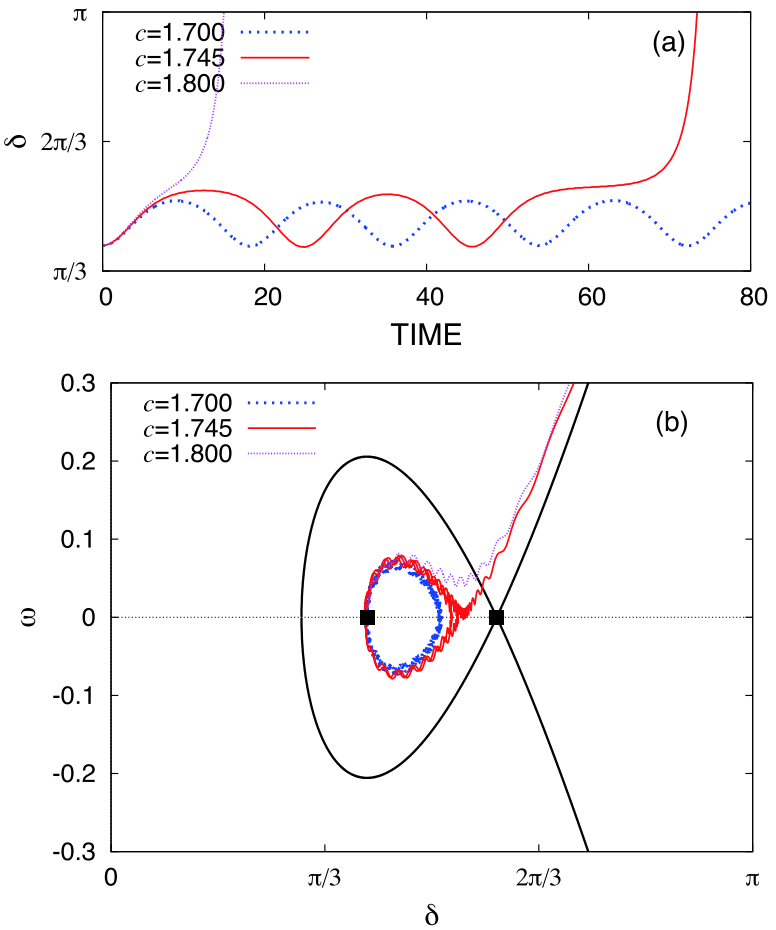

and by using the above orthonormality and (14),

$$
\sum_{j=1}^{N-1} e_{k j} \sqrt{\frac{2 I_{j}}{\Omega_{j}}} \sin \theta_{j}+e_{k N} u_{N}=\delta_{k} .
$$

Because of $e_{k N} u_{N}=\delta$, the argument of the sinusoidal function in (19) coincides with $\delta_{i}$. Equations (19) are identical to (9).

\subsection{Mode Dynamics}

Putting initial energy into any of the eigenmodes of the linear system leads to CSI, but the minimum potential energy differs with each mode. Figure 5 shows three trajectories of coupled swing dynamics for the first mode disturbance. The mode disturbance is given as the initial conditions:

$$
\left(\delta_{i}(0), \omega_{i}(0)\right)=\left(\delta_{\mathrm{c}}+c e_{i, 1}, 0\right),
$$

where $c$ is the strength of mode disturbance. The disturbance implies that at the initial time $t=0$, all the energy is in the potential energy, in the shape of the first linear mode. The left box ( $\boldsymbol{\square})$ in Fig. 5 represents the initial condition of the first mode disturbance in the $\delta-\omega$ plane. The trajectories with $c=1.745$ and 1.8 show behaviors similar to that in Fig. 4(b). They start inside the region defined by the homoclinic orbit $\Gamma_{0}$, escape from it, and diverge to infinity. This indeed shows the CSI phenomena 
caused by the first mode disturbances. Higher mode disturbances also induce CSIs, similar to that for the first mode disturbance.

Next we quantitatively investigate the dynamics of oscillatory modes. Figure 6 shows trajectories of (3) projected onto the modal variable $\left(u_{j}, v_{j}\right)$ planes. The figures are for (a) the local disturbance at $\delta_{N / 2}(0)=-0.352$ and (b) the first mode disturbance at $c=1.745$ (see (23)). It should be noted for Fig. 6(b) that there are other trajectories of oscillatory modes that do not appear here, because they have much smaller amplitudes compared to the trajectory shown above. For the dynamical system of (16) and (17), if there is no coupling between the eigenmodes, that is, $p_{\mathrm{m}}=b=0$, then the system becomes integrable, and each oscillatory mode shows a periodic motion. Figure 6 suggests that each oscillatory mode keeps its periodic motion under the presence of weak coupling. Note that figures similar to Fig. 6 are reported in Eisenhower and Mezić (2007, 2008), Du Toit et al. (2009) for other oscillatory systems.

\subsection{The Dynamical Mechanism}

Because of the above results on oscillatory modes, we now assume that the interaction of the $1, \ldots, N-1$ eigenmodes is negligible, and that their oscillations affect the collective dynamics of the nonlinear mode as external forces. This assumption was developed in Du Toit et al. (2009) for the analysis of coupled oscillators of the type which we consider here. We call the resulting theory the Harmonic Field Theory, as opposed to the well-known Mean Field Theory which would in this case correspond to averaging over fast angles and would not predict the loss of stability. Then, based on the system (19), we have the following system of collective variables:

$$
\left.\begin{array}{l}
\frac{\mathrm{d} \delta}{\mathrm{d} t}=\omega, \\
\frac{\mathrm{d} \omega}{\mathrm{d} t}=p_{\mathrm{m}}-\frac{b}{N} \sum_{i=1}^{N} \cos \left(\sum_{j=1}^{N-1} e_{i j} u_{j}(t)\right) \sin \delta-\frac{b}{N} \sum_{i=1}^{N} \sin \left(\sum_{j=1}^{N-1} e_{i j} u_{j}(t)\right) \cos \delta,
\end{array}\right\}
$$

with

$$
u_{j}(t)=c_{j} \cos \Omega_{j} t
$$

where $c_{j}$ is the amplitude of the $j$ th mode oscillation and is assumed to be constant. This approximation, when inserted in (24), implies that the collective dynamics are described by the second-order, non-autonomous system with external forces of multiple frequencies. The external forces depend on how a disturbance is added to the grid. If $c_{j}$ is zero for all $j$, that is, there is no disturbance, then the system (24) becomes the system (10) describing the motion of a single pendulum. This suggests that the approximate planar, time-dependent system (24) describes the dynamics of the full system (3) close to the two-dimensional invariant manifold $\mathcal{I}$ for small $c_{j}$.

Figure 5 demonstrates the CSI caused by a single first mode disturbance. In the following, we consider the case of a single $n$th mode disturbance, namely $c_{j}=0$ 

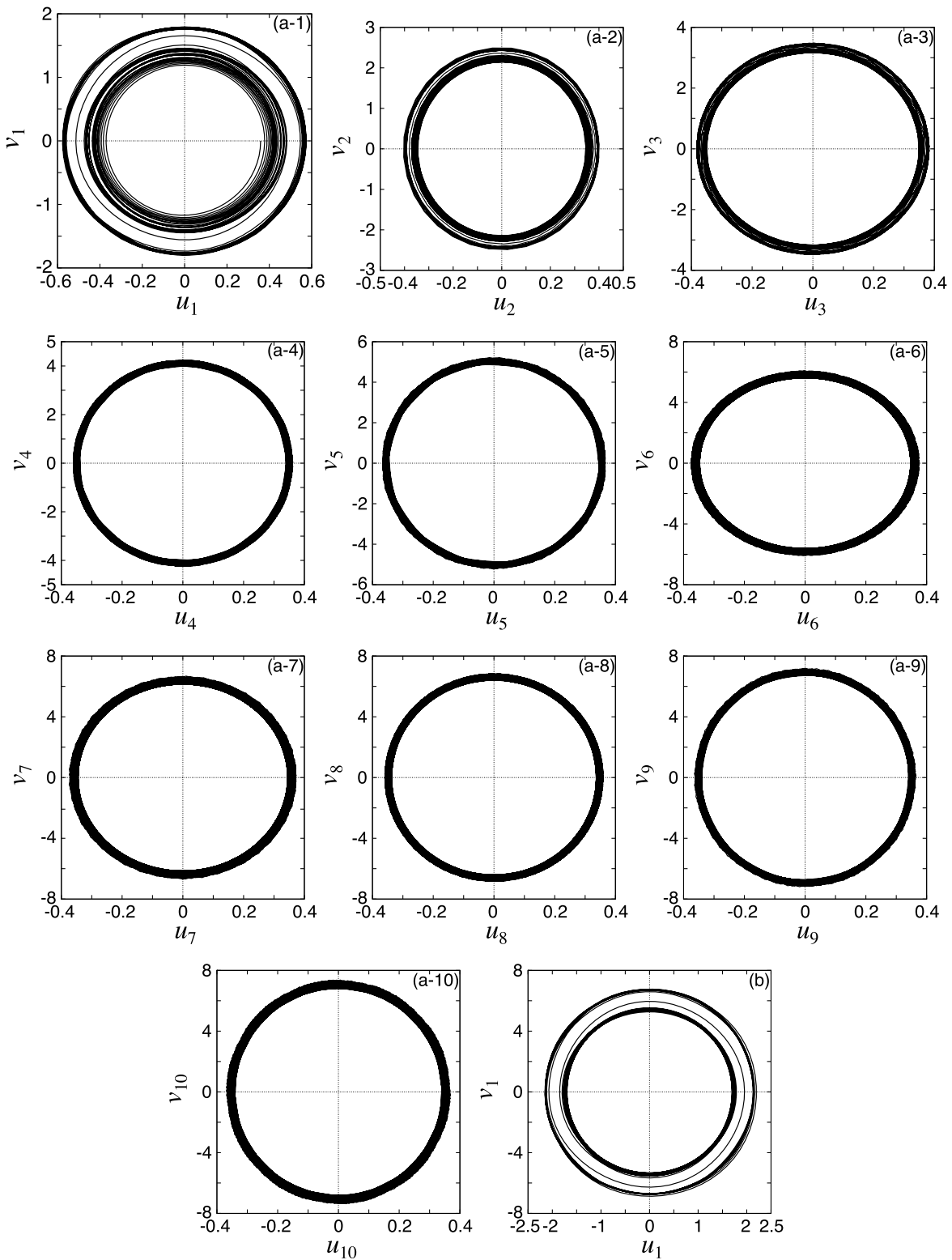

Fig. 6 Dynamics of the oscillatory modes under coherent swing instability. We show trajectories of the swing equations (3) projected onto the modal variable $\left(u_{j}, v_{j}\right)$ planes: (a) local disturbance at $\delta_{N / 2}(0)=-0.352$. For (a) the other trajectories of the oscillatory modes are omitted. (a) Local disturbance at $\delta_{N / 2}(0)=-0.352$ and (b) first mode disturbance at $c=1.745$. For (b) there are trajectories projected onto other modal planes that do not appear, because they are very small compared to the trajectory (b) 
except for $j=n$. For this case, using the standard Bessel function $J_{k}(\cdot)$, we can re-write (24) as follows:

$$
\left.\begin{array}{l}
\frac{\mathrm{d} \delta}{\mathrm{d} t}=\omega, \\
\frac{\mathrm{d} \omega}{\mathrm{d} t}=p_{\mathrm{m}}-\frac{b}{N} \sum_{i=1}^{N} J_{0}\left(e_{i n} c\right) \sin \delta-\frac{b}{N} e(t, \delta),
\end{array}\right\}
$$

where $c=c_{n}$, and $e(t, \delta)$ is the time-dependent forcing term defined by

$$
\begin{aligned}
e(t, \delta)= & 2 \sum_{i=1}^{N}\left\{\sum_{k=1}^{\infty}(-1)^{k} J_{2 k}\left(e_{i n} c\right) \cos 2 k \Omega_{n} t\right\} \sin \delta \\
& +2 \sum_{i=1}^{N}\left\{\sum_{k=0}^{\infty}(-1)^{k} J_{2 k+1}\left(e_{i n} c\right) \cos (2 k+1) \Omega_{n} t\right\} \cos \delta .
\end{aligned}
$$

This implies that the collective dynamics are described by the second-order, nonautonomous system with external forcing of period $T_{n}=2 \pi / \Omega_{n}$, with the base frequency $\Omega_{n}$, and harmonics $2 \Omega_{n}, 3 \Omega_{n} \ldots$ It is noteworthy that the critical power $b$ of the system (10) changes in the effective coefficient $b_{\text {eff }}(c)=(b / N) \sum_{i=1}^{N} J_{0}\left(e_{i n} c\right)$ of the planar system (26). If $p_{\mathrm{m}}<b_{\mathrm{eff}}(c)$, the planar system (26) without $e(t, \delta)$ has one homoclinic orbit $\Gamma_{\mathrm{c}}$ connecting the saddle-type equilibrium $\left(\delta_{\mathrm{c}}^{*}, 0\right)$ to itself:

$$
\delta_{\mathrm{c}}^{*}=\pi-\sin ^{-1} \frac{p_{\mathrm{m}}}{\frac{b}{N} \sum_{i=1}^{N} J_{0}\left(e_{i n} c\right)} .
$$

The escape criterion developed in Eisenhower and Mezić (2010) for static perturbations can now be based on the Hamiltonian of the steady part of the above system (26); it is

$$
\mathcal{H}_{n}\left(\delta_{i n}, 0 ; c\right) \geq \mathcal{H}_{n}\left(\delta_{\mathrm{c}}^{*}, 0 ; c\right),
$$

where $\delta_{\text {in }}$ is the perturbation angle, and $\mathcal{H}_{n}(\delta, \omega ; c)$ is defined as

$$
\mathcal{H}_{n}(\delta, \omega ; c)=\frac{1}{2} \omega^{2}-p_{\mathrm{m}} \delta-\frac{b}{N} \sum_{i=1}^{N} J_{0}\left(e_{i n} c\right) \cos \delta .
$$

Figure 7 shows several examples of homoclinic orbit $\Gamma_{\mathrm{c}}$ for $n=1$ and the associated collective dynamics produced by simulating the full system (3). Figures (a)-(c) plot the trajectories of coupled swing dynamics for the same parameters as those used in Fig. 5 at $b_{\text {int }}=100$ and (d)-(f) the trajectories at $b_{\text {int }}=10$. Except for (a), the trajectories escape the region defined by $\Gamma_{0}$ in the undisturbed $(c=0)$ case. The box (ם) represents the initial condition in the collective coordinate plane that is selected to be the same for all strengths of modal perturbation $c$. The homoclinic orbit $\Gamma_{\mathrm{c}}$ shrinks as the parameter $c$ increases from 1.7 to 1.8 , and it passes through the initial condition. The relationship between $\Gamma_{\mathrm{c}}$ and collective dynamics is simple in the 

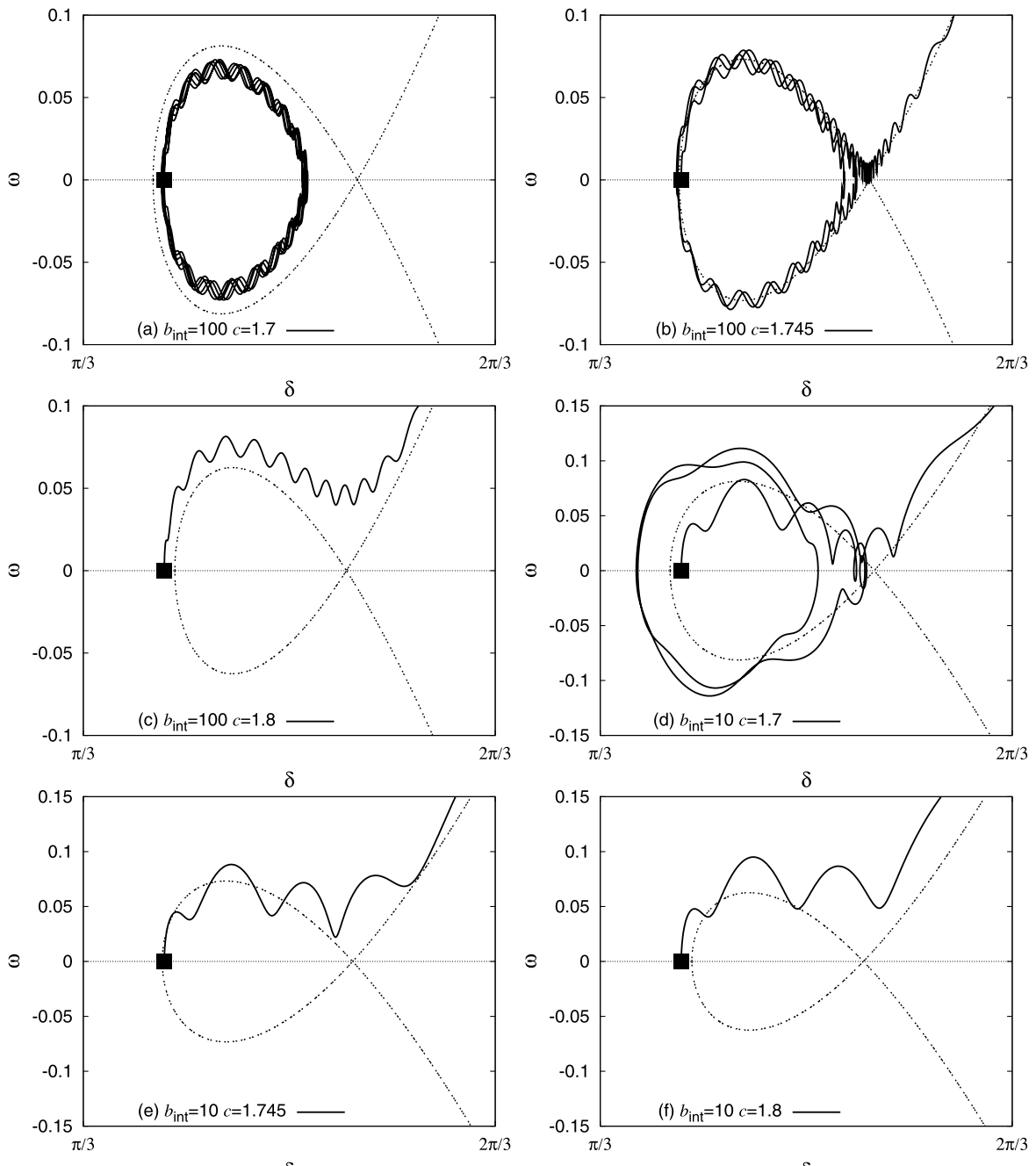

$\delta$

$\delta$

Fig. 7 Relationship between homoclinic orbit $\Gamma_{\mathrm{c}}$ and collective dynamics of coupled swings for first mode disturbance: $(\mathbf{a})-(\mathbf{c}) b_{\text {int }}=100$ and (d) $-(\mathbf{f}) b_{\text {int }}=10$

cases (a)-(c) of a large setting of parameter $b_{\text {int }}$. If the initial condition ( $\left.\mathbf{\square}\right)$ is inside $\Gamma_{\mathrm{c}}$ in Fig. 7(a), then instability does not occur. If the initial condition is outside $\Gamma_{\mathrm{c}}$ in Fig. 7(c), then CSI occurs. The relationship between the homoclinic and collective dynamics, on the other hand, is not simple in the cases (d)-(f) where $b_{\text {int }}$ is much smaller. The trajectories diverge in Figs. 7(d)-(f), not depending on where the initial condition is placed. The parameter $b_{\text {int }}$ determines the angular frequencies $\Omega_{j}$ of the linear modes between coupled machines (see (11)), and $p_{\mathrm{m}}$ and $b$ determine the timescale of the dynamics in the nonlinear mode (see (19)) as well as the strength of coupling between different linear modes (see (17)). In the case of $b_{\text {int }}=10$, the interaction between the linear modes and the local nonlinear dynamics occurs at the 
same timescales, and thus the stronger exchange of energy between the non-uniform linear modes can occur. The reduced-order system (26) is derived when we ignore the exchange of energy between such modes. This is why the phase space analysis of (26) is not valid for an analysis of CSI under $b_{\text {int }}=10$.

Now let us summarize the dynamics of CSI. The non-local instability which we study here is governed by the dynamics of the nonlinear mode and is associated with a transition across the homoclinic orbit $\Gamma_{\mathrm{c}}$. The intermediate (long) oscillation in Figs. 5(b) and 7(b) at $c=1.745$ corresponds to wandering-like motions near $\Gamma_{\mathrm{c}}$, which have been described as multi-swing motions in e.g. Chu (2005). Here the homoclinic orbit $\Gamma_{\mathrm{c}}$ depends on both the parameters $\left(p_{\mathrm{m}}, b\right)$ of the generator and the eigenfunction $\left\{e_{i n}\right\}_{i=1}^{N}$ of the loop grid. In addition, $\Gamma_{\mathrm{c}}$, shrinks with the increase of the initial perturbation $c$. This clearly shows that CSI occurs as a result of the interplay between grid topology and local dynamics. Thus, the reduced-order system (26) with $\Gamma_{\mathrm{c}}$ quantifies both the effects of grid topology and of disturbances; the former effect determines the eigenfunction of the linear oscillations, and the latter one determines the emergent escapes from the region defined by $\Gamma_{0}$.

\subsection{Remarks}

The analysis performed above is applicable to the cases of multiple-mode and local disturbances. When considering any disturbance with $N-1$ eigenmodes for the system (24), we can re-write it as follows:

$$
\left.\begin{array}{l}
\frac{\mathrm{d} \delta}{\mathrm{d} t}=\omega, \\
\frac{\mathrm{d} \omega}{\mathrm{d} t}=p_{\mathrm{m}}-b_{\mathrm{eff}}(\boldsymbol{c}) \sin \delta-\frac{b}{N} e(t, \delta),
\end{array}\right\}
$$

where $e(t, \delta)$ is the time-dependent term and has zero time average. The effective coefficient $b_{\text {eff }}(\boldsymbol{c})$ for $\boldsymbol{c}=\left(c_{1}, \ldots, c_{N-1}\right)^{\mathrm{T}}$ is defined as

$$
b_{\text {eff }}=\left\{\begin{aligned}
\frac{b}{N} \sum_{i=1}^{N}\left(\prod_{j=1}^{N / 2-1} J_{0}\left(e_{i j} c_{j}+e_{i, N-j} c_{N-j}\right)\right) J_{0}\left(e_{i, N / 2} c_{N / 2}\right) \\
\quad \text { if } N \geq 2 \text { is even, } \\
\frac{b}{N} \sum_{i=1}^{N} \prod_{j=1}^{(N-1) / 2} J_{0}\left(e_{i j} c_{j}+e_{i, N-j} c_{N-j}\right) \\
\text { if } N \geq 3 \text { is odd. }
\end{aligned}\right.
$$

The system (31) is derived in Appendix B. Thus, the general situation of multiplemode initial excitations leads to motion of a point in a sinusoidal-shape potential under excitation with different (incommensurate) frequencies $\Omega_{i}(i=1, \ldots, N-1)$. Note that each term in $e(t, \delta)$ contains at least one Bessel function $J_{k}\left(e_{i j} c_{j}\right)(k>0)$. For small $c_{j}$, the order of $b \cdot e(t, \delta) / N$ is expected to be smaller than that of the coefficient of $\sin \delta$, because the Bessel function $J_{k}(x)$ for $k>0$ converges to zero as $x \rightarrow 0$. Thus the planar system (including the case of a single $n$th mode disturbance $\left.\boldsymbol{c}=\left(c_{n}\right)\right)$ can be treated with Melnikov's method (Melnikov 1963; Guckenheimer and Holmes 1983).

The derivation of the reduced dynamical system (24) for collective variables does not depend on network properties such as topology and strength of interconnections 


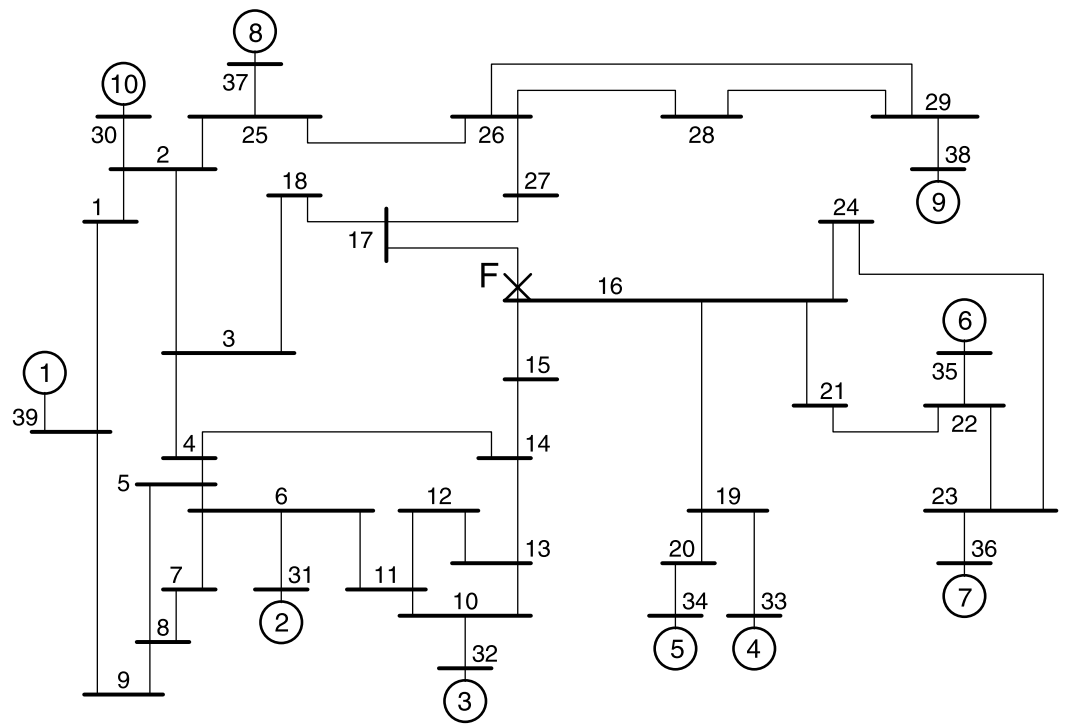

Fig. 8 The New England (NE) 39-bus test system (Athay et al. 1979; Pai 1989). The generators are denoted by circled numbers

(weight). They control the parameters of eigenvectors $e_{i j}$ and eigenfrequencies $\Omega_{j}$ only. Hence, the system (24) is applicable to a classical power grid model with an arbitrary network structure, and the phase plane analysis performed above is useful for analysis of the CSI phenomena in general power grids. This suggests that the system (24) may be used for design of power grids with stability specifications, for example, to minimize the rate of occurrence in CSI.

\section{Analysis of a Practical Test System}

In this section, we demonstrate that CSI occurs in the New England (NE) test system shown in Fig. 8. The test system consists of 10 generation units (equivalent 10 synchronous generators), 39 buses, and AC transmission lines. Most of the buses have constant active and reactive power loads. The details of the grid, such as unit rating and line data, are available in Pai (1989). In Sect. 5.1 we numerically observe the CSI phenomenon by using the swing equations. In Sect. 5.2 we investigate the phenomenon in the NE test system by using the notions of collective variables and partial linearization, and of POD.

\subsection{Numerical Simulation}

First of all, we introduce the equations of motion for the NE test system. Assume that generator 1 is the infinite bus in order to explicitly represent the outside of the system. The short-term swing dynamics of generators 2-10 are represented by the 
swing equations (Kundur 1994):

$$
\left.\begin{array}{rl}
\frac{\mathrm{d} \delta_{i}}{\mathrm{~d} t}= & \omega_{i}, \\
\frac{H_{i}}{\pi f_{\mathrm{b}}} \frac{\mathrm{d} \omega_{i}}{\mathrm{~d} t}= & -D_{i} \omega_{i}+P_{\mathrm{m} i} \\
& -G_{i i} E_{i}^{2}-\sum_{j=1, j \neq i}^{10} E_{i} E_{j}\left\{G_{i j} \cos \left(\delta_{i}-\delta_{j}\right)+B_{i j} \sin \left(\delta_{i}-\delta_{j}\right)\right\},
\end{array}\right\}
$$

where the integer label $i=2, \ldots, 10$ denotes generator $i$. The variable $\delta_{i}$ is the angular position of rotor in generator $i$ with respect to bus 1 and it is in radians [rad]. The variable $\omega_{i}$ is the deviation of rotor speed in generator $i$ relative to system angular frequency $2 \pi f_{\mathrm{b}}=2 \pi \times(60 \mathrm{~Hz})$ and is in radians per second $[\mathrm{rad} / \mathrm{s}]$. We set the variable $\delta_{1}$ to a constant, because bus 1 is assumed to be the infinite bus. The parameters $f_{\mathrm{b}}, H_{i}, D_{i}, P_{\mathrm{m} i}, E_{i}, G_{i i}, G_{i j}$, and $B_{i j}$ are in per-unit system except for $H_{i}$ and $D_{i}$ in seconds [s], and for $f_{\mathrm{b}}$ in Hertz [Hz]. The mechanical input power $P_{\mathrm{m} i}$ of generator $i$ and the internal voltage $E_{i}$ of generator $i$ are normally constant for short-term rotor angle stability (Kundur 1994). The parameter $H_{i}$ is the per-unit time inertia constant of generator $i$, and $D_{i}$ is its damping coefficient. The parameter $G_{i i}$ is the internal conductance, and $G_{i j}+\mathrm{j} B_{i j}$ (where $\mathrm{j}$ is the imaginary unit) is the transfer impedance between generators $i$ and $j$. They are the parameters that change with network topology changes. Electrical loads are simply modeled as passive impedances.

We numerically simulate coupled swing dynamics of generators 2-10. All numerical simulations discussed in this section were performed using MATLAB/Simulink: for example, the ode45 function was used for numerical integrations of (33). The voltage $E_{i}$ and the initial condition $\left(\delta_{i}(0), \omega_{i}(0)=0\right)$ for generator $i$ are fixed using power flow computation. The inertia constant $H_{i}$ is the same as in Pai (1989). For the simulation we use the following load conditions: $P_{\mathrm{m} i}$ and constant power loads are $50 \%$ at their rating. The damping $D_{i}$ is fixed at 0.005 s for each generator. ${ }^{1}$ The elements $G_{i i}, G_{i j}$, and $B_{i j}$ are calculated using the data in Pai (1989) and the result of power flow computation. We use the following fault condition: each generator operates at a steady condition at $t=0 \mathrm{~s}$. Then a three-phase fault happens at point $\mathrm{F}$ near bus 16 at $t=1 \mathrm{~s}-20 /(60 \mathrm{~Hz})=2 / 3 \mathrm{~s}$, and line $16-17$ trips at $t=1 \mathrm{~s}$. The fault duration is 20 cycles of a $60-\mathrm{Hz}$ sine wave. The fault is simulated by adding a small impedance $\left(10^{-7} \mathrm{j}\right)$ between bus 16 and the ground.

Figure 9 shows the time responses of angular position $\delta_{i}$ and relative rotor speed $\omega_{i}$ of generator $i$. Before $t=2 / 3 \mathrm{~s}$ (the onset time of fault), each generator operates at a steady condition. In the fault duration from $t=2 / 3 \mathrm{~s}$ to $1 \mathrm{~s}$, all the generators $2-10$ accelerate from their steady conditions. After the line trip at $t=1 \mathrm{~s}$, they respond in an oscillatory manner. These oscillations are bounded during the period from $t=1 \mathrm{~s}$ to $8 \mathrm{~s}$. At about time $8 \mathrm{~s}$ they begin to grow coherently. That is, every generator loses

\footnotetext{
${ }^{1}$ When the relative rotor speed $\omega_{i}$ is in per-unit system with base $2 \pi f_{\mathrm{b}}$, the damping coefficient $D_{i}=$ $0.005 \mathrm{~s}$ is equal to 1.88 in per-unit system with its base $1 /\left(2 \pi f_{\mathrm{b}}\right)$.
} 

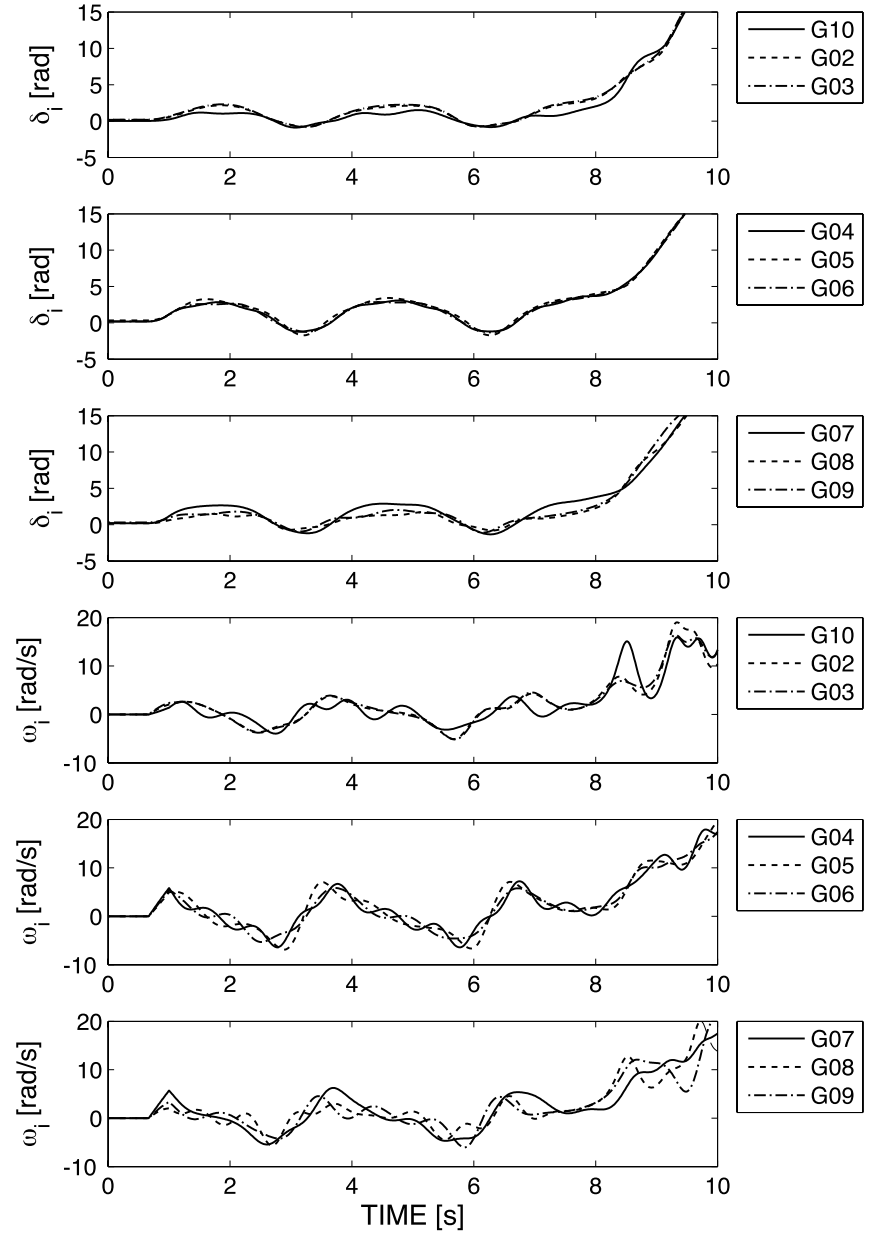

Fig. 9 Coupled swing dynamics in the swing equations (33) of the New England (NE) 39-bus test system. The upper three plots are for angular positions $\delta_{i}$ of the nine generators, and the lower plots are for the relative rotor speed $\omega_{i}$

synchronism with the infinite bus at the same time. This corresponds to the growth of the amplitude of the inter-area mode oscillation between the NE test system and the infinite bus, namely, the outside of the grid. This is typical of the CSI phenomenon.

Note that for the loop grid in Sects. 2-4, CSI exhibits a dynamical transition from inter-machine mode oscillation to the instability of the inter-area mode oscillation. The non-existence of an inter-machine mode in the NE test system is based on the model structure of the grid. The swing equations (33) have all-to-all coupling, and the equations (1) for the loop grid have nearest-neighbor coupling. The all-toall coupling — which leads to the lack of the inter-machine mode-physically comes from the model assumption and grid topology: in the NE test system, we derive the swing equations by considering transformer reactances and transient reactances in 
every generator. In addition, the graph structure of the NE test system results in the all-to-all and heterogeneous coupling structure in (33).

\subsection{Numerical Analysis}

In this section we analyze the instability phenomenon observed in the NE test system. We introduce the notion of collective variables and describe the simplification of the problem rendered by the linearization of interconnection terms, as developed in Sect. 4. These are the same tools used for analysis of the simple loop grid. Furthermore, we use the POD (Proper Orthonormal Decomposition) and obtain a set of empirical modes of the phenomenon.

\subsubsection{Collective Dynamics}

In Sect. 4 we showed that CSI involved the divergent motion in the projection of the full-system dynamics onto the phase plane of collective variables. The collective variables are known as the COA (Center-Of-Angle) variables (Athay et al. 1979). Here we discuss the relationship between the phenomena uncovered in the loop grid and observed in Fig. 9. For the NE test system, the COA $\delta_{\mathrm{COA}}$ and its time derivative $\omega_{\mathrm{COA}}$ are defined as

$$
\delta_{\mathrm{COA}}=\sum_{i=2}^{10} \frac{H_{i}}{H} \delta_{i}, \quad \omega_{\mathrm{COA}}=\frac{\mathrm{d} \delta_{\mathrm{COA}}}{\mathrm{d} t}=\sum_{i=2}^{10} \frac{H_{i}}{H} \omega_{i},
$$

where $H=\sum_{i=2}^{10} H_{i}$. The variables $\delta_{\mathrm{COA}}$ and $\omega_{\mathrm{COA}}$ describe the averaged motion of all the generators in the test system. Figure 10 plots the trajectory of (33) showing

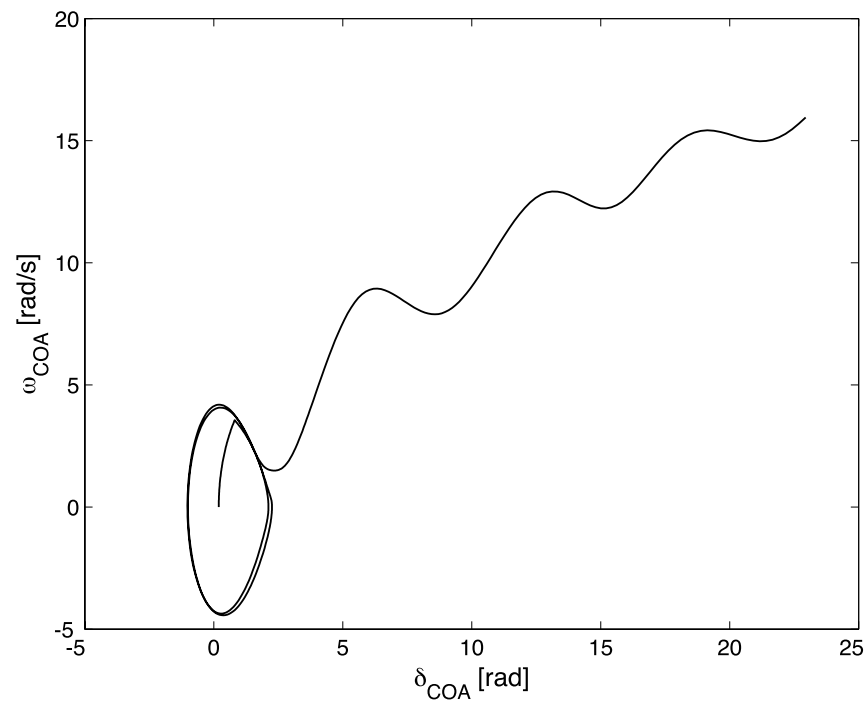

Fig. 10 Collective dynamics of the New England (NE) 39-bus test system. This plot corresponds to the dynamics of Center-Of-Angle (COA) variables for the phenomenon observed in Fig. 9 
the phenomenon in Fig. 9 in the $\delta_{\mathrm{COA}}-\omega_{\mathrm{COA}}$ plane. The trajectory starts near the origin at time $0 \mathrm{~s}$, makes a couple of almost periodic loops around the initial point and finally diverges. This behavior of collective variables is similar to that investigated in the loop grid (see Figs. 4(b) and 5(b)), in which a projected trajectory escapes the region of bounded motions due to a perturbation originating from the interconnections between individual generators.

\subsubsection{Partial Linearization}

In Sect. 4 we studied the swing equations (3) with weak local nonlinear and strong linear interconnection terms. The local term $p_{\mathrm{m}}-\sin \delta_{i}$ represents the interaction of an individual generator with the infinite bus. The interconnection term $b_{\text {int }}\left\{\left(\delta_{i-1}-\delta_{i}\right)-\left(\delta_{i}-\delta_{i+1}\right)\right\}$ represents the interaction between nearest-neighbor generators. The value of $b_{\text {int }} / p_{\mathrm{m}}$ in Sects. 3 and 4 is of order $10^{2}$. Thus, the magnitude of the interaction between any two generators is much larger than that of the interaction of an individual generator with the infinite bus. By linearizing the interconnection term in (33), we can evaluate the magnitudes of the local and interconnection terms in a way consistent with Sect. 4 . We assume that in the NE test system, the difference between any two swings $\delta_{i}(t)$ and $\delta_{j}(t)(i, j=2, \ldots, 10)$ is small: for a small parameter $\epsilon$,

$$
\delta_{i}(t)-\delta_{j}(t)=\epsilon z_{i j}(t)
$$

where $z_{i j}(t)$ is the time-dependent function containing high harmonic components. By substituting the small difference $\epsilon z_{i j}(t)$ into the interconnection term of (33), we have the following first-order approximation:

$$
\frac{H_{i}}{\pi f_{\mathrm{b}}} \frac{\mathrm{d}^{2} \delta_{i}}{\mathrm{~d} t^{2}} \approx-D_{i} \frac{\mathrm{d} \delta_{i}}{\mathrm{~d} t}+P_{i}-f_{i}\left(\delta_{i}\right)-\sum_{j=2}^{10} A_{i j} \delta_{j},
$$

where $P_{i}$ is the effective input power of generator $i$, defined as

$$
P_{i}=P_{\mathrm{m} i}-\sum_{j=2}^{10} E_{i} E_{j} G_{i j} .
$$

Also the nonlinear function $f_{i}\left(\delta_{i}\right)$ is defined as

$$
f_{i}\left(\delta_{i}\right)=E_{i} E_{1}\left\{G_{i 1} \cos \left(\delta_{i}-\delta_{1}\right)+B_{i 1} \sin \left(\delta_{i}-\delta_{1}\right)\right\} .
$$

The constant $A_{i j}$ is the element of the interconnection matrix A, defined by

$$
A_{i j}= \begin{cases}\sum_{k=2, k \neq i}^{10} E_{i} E_{k} B_{i k} & \text { if } i=j, \\ -E_{i} E_{j} B_{i j} & \text { otherwise. }\end{cases}
$$

On the right-hand side of (36), the last term, $-\sum A_{i j} \delta_{j}$, represents the interaction between generator $i$ and the other generators in the NE test system and hence the 
Table 1 Numerical result of the parameter $P_{i}$ and $L^{1}$-norm $\left\|f_{i}\right\|_{1}$ for the local effect

\begin{tabular}{lrllllllll}
\hline & $i=10$ & 2 & 3 & 4 & 5 & 6 & 7 & 8 & 9 \\
\hline$P_{i}$ & -1.1632 & 1.0024 & 1.3686 & 0.3357 & 1.1662 & 0.7714 & 0.6754 & 0.9787 & 1.8524 \\
$\left\|f_{i}\right\|_{1}$ & 11.6381 & 5.8010 & 6.1909 & 2.4759 & 0.9802 & 2.4050 & 2.0003 & 5.7453 & 4.0140 \\
\hline
\end{tabular}

interconnection (or inter-machine) effect. The other terms on the right-hand side of (36), except for the constant $P_{i}$, represent the interaction of generator $i$ with the infinite bus and hence the inter-area effect. Note that, dynamically, the inter-area terms depend only on the local angular position $\delta_{i}$. We assume that the damping coefficient $D_{i}$ is relatively small. To quantify the relative contributions of the linear interconnection and the local effects, we calculate maximum values of these terms, excluding the small damping contribution. For the estimation of interconnection effect, we use the induced 1-norm of the matrix $A$, denoted by $\|\mathrm{A}\|_{1}$ as follows:

$$
\|\mathrm{A}\|_{1}=\max _{i} 2\left|A_{i i}\right| .
$$

The induced norm of A gives a measure of how much the corresponding linear map affects the swing dynamics of generators. For the local effect, we calculate the parameter $P_{i}$ and $L^{1}$-norm of the continuous function $f_{i}\left(\delta_{i}\right)$ on the circle $\mathbb{T}^{1}$, denoted by $\left\|f_{i}\right\|_{1}$. Since $E_{i} E_{1}$ is positive, the $L^{1}$-norm of $f_{i}$ is given by

$$
\left\|f_{i}\right\|_{1}=2 E_{i} E_{1}\left\{\left(G_{i 1} \cos \delta_{1}-B_{i 1} \sin \delta_{1}\right)^{2}+\left(G_{i 1} \sin \delta_{1}+B_{i 1} \cos \delta_{1}\right)^{2}\right\}^{1 / 2} .
$$

The calculation of $\left\|f_{i}\right\|_{1}$ is given in Appendix C. Using the current parameters, the 1-norm $\|\mathrm{A}\|_{1}$ becomes 20.9311 . The parameter $P_{i}$ and $L^{1}$-norm $\left\|f_{i}\right\|_{1}$ are shown in Table 1 and are of order 1, except for $\left\|f_{10}\right\|_{1}$. The averages of $P_{i}$ and $\left\|f_{i}\right\|_{1}$ are much smaller (one-fifth) than the 1-norm $\|A\|_{1}$. Hence, in the NE test system, the magnitude of the interaction between two generators is larger than that of a generator with the infinite bus. This clearly shows that in the NE test system, we have a dynamical system with weak local and strong interconnection terms for which the CSI phenomenon is relevant. Note that the interconnection term is strong here due to the topology of the interaction of generators, where many generators affect the dynamics of any single one, as opposed to the loop grid in Sect. 4, where the strength of the interconnection between the nearest-neighbors caused the CSI phenomenon.

\subsubsection{Empirical Mode Decomposition}

In Sect. 4 we reformulated (3) by using the analytical formula of the basis vectors in the normal modes of oscillations determined by the linear interconnection term and showed that the process of CSI was decomposed into one nonlinear mode and linear oscillatory modes. For (33) and (36), it is difficult to find analytical formulas of the normal modes determined by the interconnection terms. In this section, we decompose the phenomenon in Fig. 9 using POD, which was used in the past in the context of power grids (Parrilo et al. 1999; Messina and Vittal 2007). 
POD provides a basis for the modal decomposition of an ensemble of functions, such as data obtained in the course of experiments, and it provides energy-wise, the most efficient way (however, perhaps not from a dynamical perspective (Mezić 2005a)) of capturing the dominant components of the process (Holmes et al. 1996; Feeny and Kappagantu 1998). Consider finite simulation outputs of angular positions, $\left\{\delta_{i}\left(n T_{\mathrm{s}}\right)\right\}\left(i=2, \ldots, 10, n=0, \ldots, N_{\mathrm{s}}-1\right)$ where $T_{\mathrm{s}}$ is the sampling period of outputs, and $N_{\mathrm{s}}$ is the number of samples. The outputs are represented by

$$
\delta_{i}\left(n T_{\mathrm{s}}\right)=\sum_{j=1}^{9} e_{i j} a_{j}\left(n T_{\mathrm{s}}\right)
$$

We require the time-invariant basis vectors $\left\{e_{i j}\right\}(i=2, \ldots, 10)$ to be orthonormal and closest in energy norm to the output, and call them Proper Orthonormal Modes (POMs). Every vector $\left\{e_{i j}\right\}$ is obtained by computing the correlation matrix R from $\left\{\delta_{i}\left(n T_{\mathrm{S}}\right)\right\}$ and by finding the orthonormal eigenvectors of R: see Feeny and Kappagantu (1998) for details. The time-varying coefficient $a_{j}(j=1, \ldots, 9)$ in the POD holds the following correlation property: $\left\langle a_{j} a_{k}\right\rangle=\left\langle a_{j}^{2}\right\rangle$ (if $j=k$ ) or 0 (otherwise), where $\langle\bullet\rangle$ represents a time average of $\{\bullet\}$. POMs are ordered by $\left\langle a_{j}^{2}\right\rangle \geq\left\langle a_{j+1}^{2}\right\rangle$.

POMs are obtained using $N_{\mathrm{s}}=5341$ snapshots in the simulation outputs partially shown in Fig. 9. The time interval is $\left[1 \mathrm{~s}, 90 \mathrm{~s}\right.$, and $T_{\mathrm{s}}$ is equal to $1 /(60 \mathrm{~Hz})$. Figure 11 shows the projection of the trajectory of (33) onto subspaces spanned by every POM. The projected trajectory $\left(a_{j}\left(n T_{\mathrm{S}}\right), b_{j}\left(n T_{\mathrm{S}}\right)\right)$ for the $j$ th $\operatorname{POM}(j=1, \ldots, 9)$ is computed as

$$
a_{j}\left(n T_{\mathrm{s}}\right)=\sum_{i=2}^{10} e_{i j} \delta_{i}\left(n T_{\mathrm{s}}\right), \quad b_{j}\left(n T_{\mathrm{s}}\right)=\sum_{i=2}^{10} e_{i j} \omega_{i}\left(n T_{\mathrm{s}}\right),
$$

where $n=0, \ldots, N_{\mathrm{s}}-1$ (because of the smallness of the damping term we use the same modes for the angles and their derivatives). The last two rows of Fig. 11 show the power spectra of the time series of the coefficients $a_{j}(j=1, \ldots, 9)$. In the first POM, the trajectory shows a transition from periodic motion to a divergent one. This behavior is similar to the behavior of the nonlinear mode described in Sect. 4 (see Figs. 4(b) and 5(b)). The trajectory of the first POM coincides with the trajectory projected onto the COA plane in Fig. 10 by rotating it by $180^{\circ}$ around the origin. On the other hand, in the other POMs, each trajectory shows a periodic or quasi-periodic motion. This is confirmed by looking at the results of power spectra presented in the last two rows of Fig. 11, where each power spectrum possesses one peak or only a few peaks. This modal decomposition is the same as that for the loop grid obtained in Sect. 4 (see Fig. 6). Hence we can say that the dynamic phenomenon shown in Fig. 9 results from the interaction of one non-linear mode and many linear oscillatory modes.

\subsection{Summary and Remarks}

Let us summarize the above analysis of the NE test system. The results in Sects. 5.2.1 and 5.2.3 show that for the phenomenon shown in Fig. 9, the first POM dynamics coincide with the COA dynamics. The results in Sects. 5.2.2 and 5.2.3 demonstrate that 

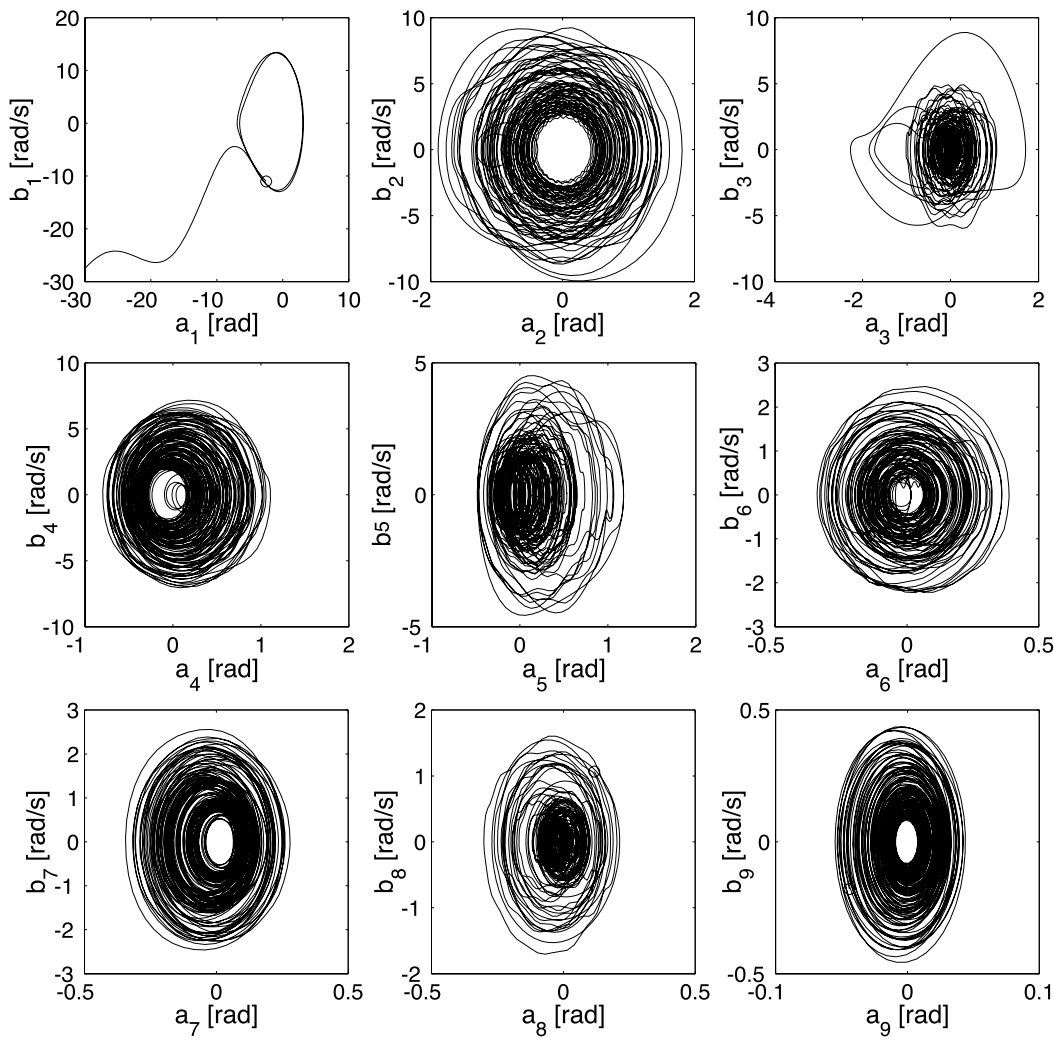

Fig. 11 Projected trajectories onto Proper Orthonormal Mode (POM) planes and power spectra of time series of coefficients $a_{j}$ during [ $1 \mathrm{~s}, 90 \mathrm{~s}$ ] with sampling frequency $60 \mathrm{~Hz}$. Zero-frequency components for the second to ninth POM are not shown here

we have the dynamical system with a nonlinear mode, which is weak compared with linear oscillatory modes. The weak nonlinear mode comes from the interaction of individual generators with the infinite bus and is determined by the nonlinear function $f_{i}\left(\delta_{i}\right)$. The strong linear modes come from the interaction between generators whose magnitude is determined by the matrix $A$ and whose magnitude is much larger than the magnitude of the nonlinear function $f_{i}\left(\delta_{i}\right)$. They act as perturbations to the weak nonlinear mode. The above dynamical structure is equivalent to that in the simple loop grid in Sects. 2-4. Thus we can say that the phenomenon shown in Fig. 9 is CSI, which is observed for the loop grid, and occurs in the dynamical system with a nonlinear mode that is weak compared to linear oscillatory modes.

The simulations performed in this section show that the CSI is robust in the sense that the phenomenon persists under additional physical refinements of the swing equations. The NE test system is an benchmark example, being a slight simplification of the real NE grid. Although the mathematical models are derived under reasonable assumptions for short-term rotor swing stability, they do not necessarily represent true dynamics of the NE grid. Here it is valuable to discuss whether the CSI in Fig. 9 can occur in a real power grid. The fault duration, which we set at 20 cycles in the 

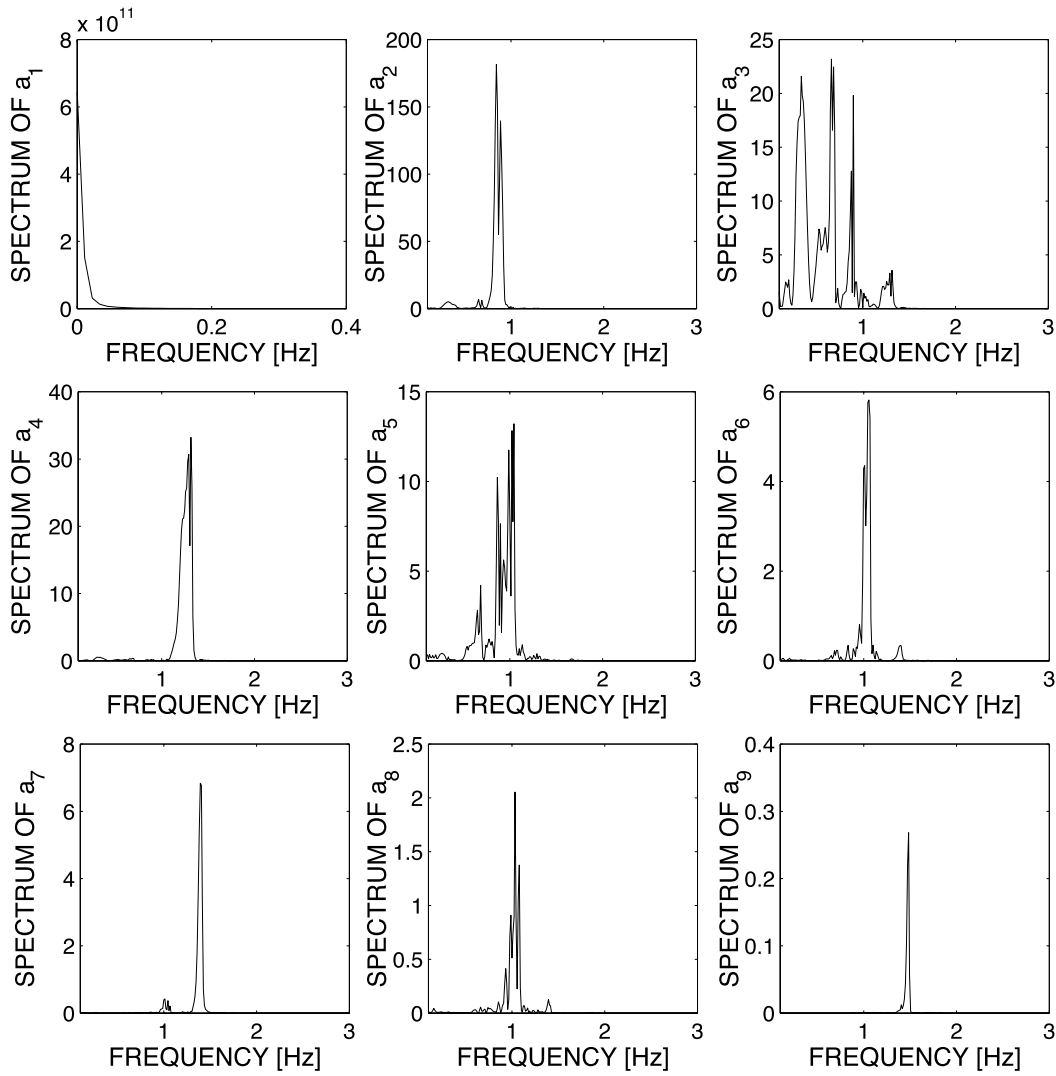

Fig. 11 (Continued)

simulations, is normally less than 10 cycles. Such a long duration may imply the malfunction of protection systems, and hence the CSI in Fig. 9 may be regarded as a rare event in short-term swing dynamics. However, in Susuki et al. (2011) we will show that in a system of interconnected power grids, CSI is observed in the case of a fault duration of less than ten cycles. Furthermore, in the simulations we ignore any effect of the load dynamics. The effect is normally negligible because it does not affect short-term swing dynamics (Kundur 1994) and will not cause any drastic change of simulation results. Thus we suggest that CSI is a phenomenon that can occur for various configurations close to real power grids.

In Susuki et al. (2009) we derived a reduced-order model for analyzing CSI using the Galerkin method. The derived model is effective for analyzing the CSI showing an immediate growth of all angular positions without any multi-swings. That model is not effective for analyzing the CSI shown in Fig. 9, because we excluded all the effects of high-order (periodic or quasi-periodic) POMs to the first POM, and the derived model fails to reproduce the transition from a periodic motion to a divergent one, observed in the first POM. Although the reduced-order model is not necessarily 
invalid for all the CSI phenomena and inter-area oscillations, the current analysis extends the validity to the case when several periodic swings are first experienced.

\section{Conclusions}

This paper was devoted to analytical and numerical analysis of CSI in the nonlinear swing equations (3) and (33). The swing equations (3) exhibit CSI of the rudimentary power grid in Fig. 1, in which after being subjected to a local disturbance, all the machines in the grid first show intermediate multi-swings (or inter-machine mode oscillations) and finally lose synchronism with the infinite bus in a coherent manner (or destabilize the inter-area mode oscillation). We developed the reduced-order, planar system (26) that describes the behavior of the nonlinear mode and characterizes the CSI. This model provides a dynamical mechanism of CSI: it is related to the escape from a potential well, or more precisely, to exit across a separatrix in the dynamical system for the amplitude of the weak nonlinear mode. The escape phenomenon can be analytically evaluated using phase plane analysis of the reduced-order system (26). Hence we successfully explain the CSI phenomenon by using (26) and regard it as a minimal mathematical model of CSI. One contribution of this paper was to develop the model that not only exhibits CSI but also explains its dynamics as the compound and global phenomenon of oscillations in the local plant mode, the multiswing (inter-machine) mode, and the inter-area mode. In addition, we show the CSI phenomenon in the New England (NE) test system. The other contribution of this paper was to demonstrate that CSI is an instability phenomenon that can occur for various realistic power grids.

As mentioned in Sect. 1, there are numerous references of instability phenomena related to CSI: inter-area mode and multi-swing instabilities. Again we comment the relationship between these and our studies. Inter-area mode oscillation is studied as modal interaction via nonlinear quadratic terms in Tamura and Yorino (1987), Vittal et al. (1991), Lin et al. (1996) and by parameter change in Dobson et al. (2001). These studies are for oscillatory instability of a group of generators, that is, development of inter-area mode oscillation. Our result pertains to the separation or loss of synchronism of a group of generators, that is, the divergence of inter-area mode oscillation. The divergence is not local in the sense that it does not happen due to an infinitesimally small perturbation around an equilibrium. Hence, the mechanism behind it is different from the modal interaction developed in Tamura and Yorino (1987), Vittal et al. (1991), Lin et al. (1996) and local bifurcation of stable equilibria (Dobson et al. 2001). Next, multi-swing instability has been characterized as the effect of voltage control to cause an unstable limit cycle (Vournas et al. 1996) and a tangled separatrix (Chu 2005), and interactions of inter-area and local plant modes (IEEE/CIGRE Joint Task Force on Stability Terms and Definitions 2004). Our mechanism is an exit across the separatrix, akin to Vournas et al. (1996), Chu (2005); however, it is more complicated and is obtained for multi-machine power grids. Our result analytically gives a novel dynamical perspective of the multi-swing instability, which has been recognized as the modal interaction (IEEE/CIGRE Joint Task Force on Stability Terms and Definitions 2004). 
Many follow-up studies to the present one are possible. One is to apply the developed theory to the dynamics of general nonlinear swing equations and to the cascading dynamics in real power grids, as mentioned in Sect. 1. This is partly performed in Sect. 5. Another is to explore the dynamics of small-scale electricity grids with the current approach. The condition of a strong linear network and weak local nonlinearity holds in the cases that grid components are connected via short transmission line, that their rating is smaller than the capacity of grid, and that they operate onto a large-scale power grid via a long transmission line. These can be indeed found in emergent technologies of electricity grid, for example, windfarms and microgrids. A windfarm has many small and closely coupled induction generators that operate in parallel and far from the heavily loaded area. This is exactly the same network configuration as that in this paper. A microgrid is an autonomous small-scale grid based on dispersed generation and control architecture, and it has many power sources operating in parallel. If many small grid components, including individual generation units and small grid itself, are connected under synchronization based on power electronics equipment (see e.g. Blaabjerg et al. 2006; Hikihara et al. 2007), then they may behave like the small synchronous generators in this paper. Of course, additional features of small-scale grids need to be included in the model description, because we cannot ignore the presence of transmission conductance (dissipation) and power electronics equipment that may play a role in avoiding coherent swings of rotating machines. Our analysis in this paper, however, does show that small-scale electricity grids may possess an inherent instability differing from those of large-scale, sparse power grids.

Acknowledgements We are grateful to Professor Petar Kokotović and Professor Vijay Vittal for discussions and useful suggestions. We are also grateful to Professor Yueheng Lan and Dr. Bryan Eisenhower for their careful reading of this manuscript, and to anonymous reviewers for their critical reading of the manuscript and valuable comments. This research is supported in part by JSPS Postdoctoral Fellowships for Research Abroad, Grant-in-Aid for Global COE Program "Education and Research on Photonics and Electronics Science and Engineering” from MEXT, Japan, and NICT Project ICE-IT (Integrated Technology of Information, Communications, and Energy).

Open Access This article is distributed under the terms of the Creative Commons Attribution Noncommercial License which permits any noncommercial use, distribution, and reproduction in any medium, provided the original author(s) and source are credited.

\section{Appendix A: Dimensional Analysis for Swing Equations (1)}

In this section we derive the non-dimensional swing equations (1). Under the assumptions (i)-(v) in Sect. 2, the short-term dynamics of generator $i$ are represented by the nonlinear swing equations (Kundur 1994):

$$
\frac{2 H P_{\mathrm{r}}}{\omega_{\mathrm{b}}} \frac{\mathrm{d}^{2} \delta_{i}}{\mathrm{~d} t^{2}}=P_{\mathrm{m}}-P_{\mathrm{e} i}\left(\delta_{1}, \ldots, \delta_{N}\right),
$$

where $i=1, \ldots, N$, and $N$ is the number of identical generators in the loop network. The variable $\delta_{i}$ is the angular position of rotor with respect to the infinite bus of generators labeled with integer values. The mechanical input power $P_{\mathrm{m}}$ to each 
generator in Watts [W] is assumed to be constant for short-term rotor swing stability analysis (Kundur 1994). The electrical output $P_{\mathrm{e} i}$ of the generator $i$ in Watts [W] is a function of the angular positions $\delta_{i}$. The parameters $H, P_{\mathrm{r}}$, and $\omega_{\mathrm{b}}$ are constant and are determined by grid and machine settings. The parameter $H$ is the per-unit time constant of the generator in seconds [s], $P_{\mathrm{r}}$ its the rated active power in Watts [W], and $\omega_{\mathrm{b}}$ the nominal system frequency in radians per second $[\mathrm{rad} / \mathrm{s}]$. Now, using the non-dimensional time $t^{*}=t \sqrt{\omega_{\mathrm{b}} /(2 H)}$, we have

$$
\frac{\mathrm{d}^{2} \delta_{i}}{\mathrm{~d}\left(t^{*}\right)^{2}}=\frac{P_{\mathrm{m}}}{P_{\mathrm{r}}}-\frac{1}{P_{\mathrm{r}}} P_{\mathrm{e} i}\left(\delta_{1}, \ldots, \delta_{N}\right) .
$$

The last term on the right-hand side corresponds to the non-dimensional electrical output, represented by

$$
\frac{1}{P_{\mathrm{r}}} P_{\mathrm{e} i}\left(\delta_{1}, \ldots, \delta_{N}\right)=\frac{V^{*} V_{\mathrm{inf}}^{*}}{X_{\mathrm{inf}}^{*}} \sin \delta_{i}+\frac{V^{* 2}}{X_{\mathrm{int}}^{*}}\left\{\sin \left(\delta_{i}-\delta_{i-1}\right)+\sin \left(\delta_{i}-\delta_{i+1}\right)\right\} .
$$

The first-term on the right-hand side represents the transfer of active power between generator $i$ and the infinite bus, and the second and third terms the transfer of active power between two generators. The parameters $V^{*}, V_{\text {inf }}^{*}, X_{\text {inf }}^{*}$, and $X_{\text {int }}^{*}$ are constant and in per-unit system. The constant $V^{*}$ is the terminal voltage of a generator, $V_{\text {inf }}^{*}$ the voltage of the infinite bus, $X_{\text {inf }}^{*}$ the impedance of AC transmission lines joining a generator and the infinite bus, and $X_{\text {int }}^{*}$ the impedance of AC transmission lines joining generators $i$ and $i+1$ in the loop network. We now denote by $p_{\mathrm{m}}$ the non-dimensional mechanical input power $P_{\mathrm{m}} / P_{\mathrm{r}}$, by $b$ the non-dimensional maximum power transfer $V^{*} V_{\text {inf }}^{*} / X_{\text {inf }}^{*}$ to the infinite bus, and by $b_{\text {int }}$ the non-dimensional maximum power transfer $V^{* 2} / X_{\text {int }}^{*}$ between two generators in the loop network. Thus, using the nondimensional rotor speed difference $\omega_{i}^{*}=\mathrm{d} \delta_{i}^{*} / \mathrm{d} t^{*}$ and removing $*$ from the variables, we obtain the non-dimensional swing equations (1) with the four characteristic parameters $p_{\mathrm{m}}, b, b_{\mathrm{int}}$, and $N$. The parameter $b$ becomes unity if we assume that the rated active power $P_{\mathrm{r}}$ of the generator is identical to the maximum power transfer to the infinite bus. The value of $b=1$ is used in Sects. 3 and 4 .

\section{Appendix B: Derivation of Reduced-Order System (31)}

We derive the reduced-order system (31) for any disturbance with $N-1$ eigenmodes. Recall that the set of $N-1$ eigenfrequencies, $\left\{\Omega_{j}\right\}_{j=1}^{N-1}$, obey the property $\Omega_{j}=$ $\Omega_{j-N}$ for $j=1, \ldots, N / 2-1$ (if $N \geq 2$ is even) or for $j=1, \ldots,(N-1) / 2$ (if $N \geq 3$ is odd). Then, we have

$$
\sum_{j=1}^{N-1} e_{i j} c_{j} \cos \Omega_{j} t=\left\{\begin{array}{l}
\sum_{j=1}^{N / 2-1} \tilde{c}_{j} \cos \Omega_{j} t+e_{i, N / 2} c_{N / 2} \cos \Omega_{N / 2} t \\
\text { if } N \geq 2 \text { is even, } \\
\sum_{j=1}^{(N-1) / 2} \tilde{c}_{j} \cos \Omega_{j} t \\
\text { if } N \geq 3 \text { is odd, }
\end{array}\right.
$$


where $\tilde{c}_{j}=e_{i j} c_{j}+e_{i, N-j} c_{N-j}$ for $j \neq N / 2$ and $\tilde{c}_{j}=e_{i, N / 2} c_{N / 2}$ for $j=N / 2$. By defining the new time-dependent variable $\phi_{n}(t)$ by

$$
\phi_{n}(t)=\sum_{j=n}^{N-1} e_{i j} c_{j} \cos \Omega_{j} t
$$

we have the following formula:

$$
\left(\begin{array}{l}
\sin \phi_{1}(t) \\
\cos \phi_{1}(t)
\end{array}\right)= \begin{cases}\left(\prod_{j=1}^{N / 2-1} \mathrm{~A}_{j}(t)\right) \mathrm{A}_{N / 2}(t)\left(\begin{array}{l}
0 \\
1
\end{array}\right) & \text { if } N \geq 2 \text { is even } \\
\left(\prod_{j=1}^{(N-1) / 2} \mathrm{~A}_{j}(t)\right)\left(\begin{array}{l}
0 \\
1
\end{array}\right) & \text { if } N \geq 3 \text { is odd }\end{cases}
$$

where the time-dependent matrix $\mathrm{A}_{j}(t)$ belongs to $\mathrm{SO}(2)$ and is given by

$$
\mathrm{A}_{j}(t)=\left(\begin{array}{cc}
\cos \left(\tilde{c}_{j} \cos \Omega_{j} t\right) & \sin \left(\tilde{c}_{j} \cos \Omega_{j} t\right) \\
-\sin \left(\tilde{c}_{j} \cos \Omega_{j} t\right) & \cos \left(\tilde{c}_{j} \cos \Omega_{j} t\right)
\end{array}\right)
$$

Here we recall the Jacobi-Anger expansion for the Bessel function $J_{k}(\cdot)$, namely

$$
\left.\begin{array}{l}
\cos \left(\tilde{c}_{j} \cos \Omega_{j} t\right)=J_{0}\left(\tilde{c}_{j}\right)+2 \sum_{k=1}^{\infty}(-1)^{k} J_{2 k}\left(\tilde{c}_{j}\right) \cos 2 k \Omega_{j} t, \\
\sin \left(\tilde{c}_{j} \cos \Omega_{j} t\right)=-2 \sum_{k=1}^{\infty}(-1)^{k} J_{2 k-1}\left(\tilde{c}_{j}\right) \cos (2 k-1) \Omega_{j} t .
\end{array}\right\}
$$

Here, since the $N-1$ eigenfrequencies $\Omega_{j}$ are incommensurate, by using in (49) the sum and difference formulas of the trigonometric functions, we see that the time average of $\sin \phi_{1}(t)$ over infinite time is identically zero, i.e.

$$
\begin{aligned}
\left\langle\sin \phi_{1}(t)\right\rangle & =\lim _{\tau \rightarrow \infty} \frac{1}{\tau} \int_{0}^{\tau} \sin \phi_{1}(t) \mathrm{d} t \\
& =0 .
\end{aligned}
$$

On the other hand, the time average of $\cos \phi_{1}(t)$ is generally non-zero and is given by

$$
\left\langle\cos \phi_{1}(t)\right\rangle= \begin{cases}\left(\prod_{j=1}^{N / 2-1} J_{0}\left(\tilde{c}_{j}\right)\right) J_{0}\left(\tilde{c}_{N / 2}\right) & \text { if } N \geq 2 \text { is even } \\ \prod_{j=1}^{(N-1) / 2} J_{0}\left(\tilde{c}_{j}\right) & \text { if } N \geq 3 \text { is odd. }\end{cases}
$$

Thus we have the effective coefficient $b_{\text {eff }}(\boldsymbol{c})$ in the system (31), defined as

$$
b_{\mathrm{eff}}(\boldsymbol{c})=\frac{b}{N} \sum_{i=1}^{N}\left\langle\cos \phi_{1}(t)\right\rangle
$$


where $c=\left(c_{1}, \ldots, c_{N-1}\right)^{\mathrm{T}}$. In addition to this, we obtain the concrete formula of $e(t, \delta)$ as

$$
e(t, \delta)=\sum_{i=1}^{N}\left(\cos \phi_{1}(t)-\left\langle\cos \phi_{1}(t)\right\rangle\right) \sin \delta+\sum_{i=1}^{N} \sin \phi_{1}(t) \cos \delta .
$$

By the construction of $e(t, \delta)$, we have

$$
\langle e(t, \delta)\rangle=0 .
$$

Each term of $e(t, \delta)$ contains at least one Bessel function $J_{k}\left(\tilde{c}_{j}\right)$ (for $k>1$ and some $j$ ), because of the formula (49) and the Jacobi-Anger expansion. Thus we have the reduced-order system (31).

\section{Appendix C: Calculation of $L^{\mathbf{1}}$-Norm of $f_{i}$}

The $L^{1}$-norm of the function $f_{i}\left(\delta_{i}\right)$ defined on $\mathbb{T}^{1}$ is calculated as follows:

$$
\begin{aligned}
\left\|f_{i}\right\|_{1}= & \int_{-\pi}^{+\pi}\left|f_{i}\left(\delta_{i}\right)\right| \mathrm{d} \delta_{i} \\
= & E_{i} E_{1} \int_{-\pi}^{\pi}\left|G_{i 1} \cos \left(\delta_{i}-\delta_{1}\right)+B_{i 1} \sin \left(\delta_{i}-\delta_{1}\right)\right| \mathrm{d} \delta_{i} \\
= & E_{i} E_{1} \int_{-\pi}^{\pi} \mid\left(G_{i 1} \cos \delta_{1}-B_{i 1} \sin \delta_{1}\right) \cos \delta_{i} \\
& +\left(G_{i 1} \sin \delta_{1}+B_{i 1} \cos \delta_{1}\right) \sin \delta_{i} \mid \mathrm{d} \delta_{i} \\
= & E_{i} E_{1}\left\{\left(G_{i 1} \cos \delta_{1}-B_{i 1} \sin \delta_{1}\right)^{2}\right. \\
& \left.+\left(G_{i 1} \sin \delta_{1}+B_{i 1} \cos \delta_{1}\right)^{2}\right\}^{1 / 2} \int_{-\pi}^{+\pi}\left|\sin \left(\delta_{i}+\alpha_{i}\right)\right| \mathrm{d} \delta_{i} \\
= & E_{i} E_{1}\left\{\left(G_{i 1} \cos \delta_{1}-B_{i 1} \sin \delta_{1}\right)^{2}\right. \\
& \left.+\left(G_{i 1} \sin \delta_{1}+B_{i 1} \cos \delta_{1}\right)^{2}\right\}^{1 / 2} \cdot 2,
\end{aligned}
$$

where

$$
\tan \alpha_{i}=\frac{G_{i 1} \cos \delta_{1}-B_{i 1} \sin \delta_{1}}{G_{i 1} \sin \delta_{1}+B_{i 1} \cos \delta_{1}}
$$

\section{References}

Abed, E.H., Varaiya, P.P.: Nonlinear oscillations in power systems. Electr. Power Energy Syst. 6(1), 37-43 (1984)

Andersson, G., Donalek, P., Farmer, R., Hatziargyriou, N., Kamwa, I., Kundur, P., Martins, N., Paserba, J., Pourbeik, P., Sanchez-Gasca, J., Schulz, R., Stankovic, A., Taylor, C., Vittal, V.: Causes of the 2003 major grid blackouts in North America and Europe, and recommended means to improve system dynamic performance. IEEE Trans. Power Syst. 20(4), 1922-1928 (2005) 
Arnold, V.I.: Mathematical Methods of Classical Mechanics, 2nd edn. Graduate Texts in Mathematics, vol. 60. Springer, New York (1989)

Athay, T., Podmore, R., Virmani, S.: A practical method for the direct analysis of transient stability. IEEE Trans. Power Appar. Syst. PAS-98(2), 573-584 (1979)

Avramović, B., Kokotović, P.V., Winkelman, J.R., Chow, J.H.: Area decomposition for electromechanical models of power systems. Automatica 16, 637-648 (1980)

Blaabjerg, F., Teodorescu, R., Liserre, M., Timbus, A.V.: Overview of control and grid synchronization for distributed power generation systems. IEEE Trans. Ind. Electron. 53(5), 1398-1409 (2006)

Chiang, H.-D.: Power system stability. In: Webster, J.G. (ed.) Wiley Encyclopedia of Electrical and Electronics Engineering, pp. 105-137. Wiley, New York (1999)

Chu, C.-C.: Towards a theory of multi-swing transient instability problems in electric power systems. IEICE Trans. Fundam. Electron. Commun. Comput. Sci. E88-A(10), 2692-2695 (2005)

Corsi, S., Sabelli, C.: General blackout in Italy Sunday September 28, 2003, h. 03:28:00. In: Proceedings of the IEEE PES General Meeting, Denver, USA, June 2004, vol. 2, pp. 1691-1702 (2004)

Dobson, I., Zhang, J., Greene, S., Engdahl, H., Sauer, P.W.: Is strong model resonance a precursor to power system oscillations? IEEE Trans. Circuits Syst. I, Fundam. Theory Appl. 48(3), 614-622 (2001)

Du Toit, P., Mezić, I., Marsden, J.: Coupled oscillator models with no scale separation. Physica D 238(5), 490-501 (2009)

Eisenhower, B., Mezić, I.: A mechanism for energy transfer leading to conformation change in networked nonlinear systems. In: Proceedings of the 46th IEEE Conference on Decision and Control, New Orleans, USA, December, pp. 3976-3981 (2007)

Eisenhower, B., Mezić, I.: Actuation requirements of high dimensional oscillator systems. In: Proceedings of the 2008 American Control Conference, Seattle, USA, June, pp. 177-182 (2008)

Eisenhower, B., Mezić, I.: Targeted activation in deterministic and stochastic systems. Phys. Rev. E 81, 026603 (2010)

Feeny, B.F., Kappagantu, B.: On the physical interpretation of proper orthogonal modes in vibrations. J. Sound Vib. 211(4), 607-616 (1998)

Forest, M.G., Goedde, C.G., Sinha, A.: Instability-driven energy transport in near-integrable, many degrees-of-freedom, Hamiltonian systems. Phys. Rev. Lett. 68(18), 2722-2725 (1992)

Guckenheimer, J., Holmes, P.: Nonlinear Oscillations, Dynamical Systems, and Bifurcations of Vector Fields. Applied Mathematical Sciences, vol. 42. Springer, New York (1983)

Hatziargyriou, N., Asano, H., Iravani, R., Marnay, C.: Microgrids. IEEE Power Energy Mag. 5(4), 78-94 (2007)

Hikihara, T., Sawada, T., Funaki, T.: Enhanced entrainment of synchronous inverters for distributed power sources. IEICE Trans. Fundam. Electron. Commun. Comput. Sci. E90-A(11), 2516-2525 (2007)

Holmes, P., Lumley, J.L., Berkooz, G.: Turbulence, Coherent Structures, Dynamical Systems, and Symmetry. Cambridge University Press, Cambridge (1996)

IEEE/CIGRE Joint Task Force on Stability Terms and Definitions: Definition and classification of power system stability. IEEE Trans. Power Syst. 19(2), 1387-1401 (2004)

Kopell, N., Washburn, R.B. Jr.: Chaotic motions in the two-degree-of-freedom swing equations. IEEE Trans. Circuits Syst. CAS-29(11), 738-746 (1982)

Kundur, P.: Power System Stability and Control. McGraw-Hill, New York (1994)

Lasseter, R.H., Paigi, P.: Microgrid: A conceptual solution. In: Proceedings of the IEEE 35th Power Electronics Specialists Conference, Aachen, Germany, June 20-25, pp. 4285-4290 (2004)

Lin, C.-M., Vittal, V., Kliemann, W., Fouad, A.A.: Investigation of modal interaction and its effects on control performance in stressed using normal forms of vector fields. IEEE Trans. Power Syst. 11(2), $781-787$ (1996)

Melnikov, V.K.: On the stability of the center for time periodic perturbations. Trans. Mosc. Math. Soc. 12, $1-56$ (1963)

Messina, A.R., Vittal, V.: Extraction of dynamic patterns from wide-area measurements using empirical orthogonal functions. IEEE Trans. Power Syst. 22(2), 682-692 (2007)

Mezić, I.: Spectral properties of dynamical systems, model reduction and decompositions. Nonlinear Dyn. 41, 309-325 (2005a)

Mezić, I.: Dynamics and control of large-scale molecular motion. In: Proceedings of the IFAC World Congress (2005b)

Mezić, I.: On the dynamics of molecular conformation. Proc. Natl. Acad. Sci. USA 103(20), 7542-7547 (2006)

Null, J., Archer, C.: Wind power: The ultimate renewable energy source. In: WeatherWise, July/August, pp. 34-40 (2008) 
Pai, M.A.: Energy Function Analysis for Power System Stability. Kluwer Academic, Dordrecht (1989)

Parrilo, P.A., Lall, S., Paganini, F., Verghese, G.C., Lesieutre, B.C., Marsden, J.E.: Model reduction for analysis of cascading failures in power systems. In: Proceedings of the American Control Conference, San Diego, June, pp. 4208-4212 (1999)

Peponides, G., Kokotović, P.V., Chow, J.H.: Singular perturbations and time scales in nonlinear models of power systems. IEEE Trans. Circuits Syst. CAS-29(11), 758-766 (1982)

Salam, F.M.A., Marsden, J.E., Varaiya, P.P.: Arnold diffusion in the swing equations of a power system. IEEE Trans. Circuits Syst. CAS-31(8), 673-688 (1984)

Susuki, Y., Mezić, I., Hikihara, T.: Global swing instability of multimachine power systems. In: Proceedings of the 47th IEEE Conference on Decision and Control, Cancun, Mexico, December 9-11, pp. 2487-2492 (2008)

Susuki, Y., Mezić, I., Hikihara, T.: Global swing instability in the New England power grid model. In: Proceedings of the 2009 American Control Conference, St. Luis, United States, June 10-12, pp. 3446-3451 (2009)

Susuki, Y., Mezić, I., Hikihara, T.: Coherent swing instability of power systems and cascading failures. In: 2010 IEEE Power \& Energy Society General Meeting, Minneapolis, United States, June 25-29 (2010)

Susuki, Y., Mezić, I., Hikihara, T.: Coherent swing instability of interconnected power systems and a mechanism of cascading failures (2011, in preparation)

Tamura, Y., Yorino, N.: Possibility of auto- \& hetero-parametric resonances in power systems and their relationship with long-term dynamics. IEEE Trans. Power Syst. PWRS-2(4), 890-896 (1987)

Thompson, J.M.T., Stewart, H.B.: Nonlinear Dynamics and Chaos, 2nd edn. Wiley, Chichester (2002)

Ueda, Y., Enomoto, T., Stewart, H.B.: Chaotic transients and fractal structures governing coupled swing dynamics. In: Kim, J.H., Stringer, J. (eds.) Applied Chaos. Wiley, London (1992). Chap. 8

Varghese, M., Thorp, J.S.: An analysis of truncated fractal growths in the stability boundaries of three-node swing equation. IEEE Trans. Circuits Syst. 35(7), 825-834 (1988)

Vittal, V., Bhatia, N., Fouad, A.A.: Analysis of the inter-area mode phenomenon in power systems following large disturbance. IEEE Trans. Power Syst. 6(4), 1515-1521 (1991)

Vournas, C.D., Pai, M.A., Sauer, P.W.: The effect of automatic voltage regulation on the bifurcation evolution in power systems. IEEE Trans. Power Syst. 11(4), 1683-1688 (1996)

Wiggins, S.: Global Bifurcations and Chaos: Analytical Methods. Applied Mathematical Sciences, vol. 73. Springer, New York (1988)

Wiggins, S.: Chaotic Transport in Dynamical Systems. Interdisciplinary Applied Mathematics, vol. 2. Springer, New York (1992)

Yoshida, H.: Construction of higher order symplectic integrators. Phys. Lett. A 150(5-7), 262-268 (1990) 